\title{
Improved Butterfly Optimizer-Configured Extreme Learning Machine for Fault Diagnosis
}

\author{
Helong Yu (iD, ${ }^{1}$ Kang Yuan, ${ }^{1}$ Wenshu Li, ${ }^{1}$ Nannan Zhao, ${ }^{1}$ Weibin Chen $\left(\mathbb{D}^{2}{ }^{2}\right.$ \\ Changcheng Huang $\mathbb{C}^{2},{ }^{2}$ Huiling Chen $\mathbb{C}^{,},{ }^{2}$ and Mingjing Wang $\mathbb{\circledR}^{3}$ \\ ${ }^{1}$ College of Information Technology, Jilin Agricultural University, Changchun 130118, China \\ ${ }^{2}$ College of Computer Science and Artificial Intelligence, Wenzhou University, Wenzhou, Zhejiang 325035, China \\ ${ }^{3}$ Institute of Research and Development, Duy Tan University, Da Nang 550000, Vietnam \\ Correspondence should be addressed to Weibin Chen; sun@wzu.edu.cn, Changcheng Huang; cchuang@126.com, and \\ Huiling Chen; chenhuiling.jlu@gmail.com
}

Received 18 June 2020; Revised 27 November 2020; Accepted 15 January 2021; Published 8 February 2021

Academic Editor: Narayanan Kumarappan

Copyright (c) 2021 Helong Yu et al. This is an open access article distributed under the Creative Commons Attribution License, which permits unrestricted use, distribution, and reproduction in any medium, provided the original work is properly cited.

\begin{abstract}
An efficient intelligent fault diagnosis model was proposed in this paper to timely and accurately offer a dependable basis for identifying the rolling bearing condition in the actual production application. The model is mainly based on an improved butterfly optimizer algorithm- (BOA-) optimized kernel extreme learning machine (KELM) model. Firstly, the roller bearing's vibration signals in the four states that contain normal state, outer race failure, inner race failure, and rolling ball failure are decomposed into several intrinsic mode functions (IMFs) using the complete ensemble empirical mode decomposition based on adaptive noise (CEEMDAN). Then, the amplitude energy entropies of IMFs are designated as the features of the rolling bearing. In order to eliminate redundant features, a random forest was used to receive the contributions of features to the accuracy of results, and subsets of features were set up by removing one feature in the descending order, using the classification accuracy of the SBOAKELM model as the criterion to obtain the optimal feature subset. The salp swarm algorithm (SSA) was introduced to BOA to improve optimization ability, obtain optimal KELM parameters, and avoid the BOA deteriorating into local optimization. Finally, an optimal SBOA-KELM model was constructed for the identification of rolling bearings. In the experiment, SBOA was validated against ten other competitive optimization algorithms on 30 IEEE CEC2017 benchmark functions. The experimental results validated that the SBOA was evident over existing algorithms for most function problems. SBOA-KELM employed for diagnosing the fault diagnosis of rolling bearings obtained improved classification performance and higher stability. Therefore, the proposed SBOA-KELM model can be effectively used to diagnose faults of rolling bearings.
\end{abstract}

\section{Introduction}

As a core component of mechanical, the rolling bearings are widely used in rotating machinery types such as wind turbines, aeroengines, ships, and automobiles. However, it is a considerable probability of a mechanical failure due to a bearing failure. Bearings cause failures in rotating machinery that is over $30 \%$. There are various faults of rolling bearing, including outer race, inner race, and ball, in general due to a long-term complex environment. When those faults get serious, they may cause a sudden breakdown of the machine, even the entire system, leading to substantial financial losses, and even cause casualties among workers. Overmaintenance of rolling bearings can lead to increased corporate maintenance costs. However, insufficient maintenance can easily lead to unexpected production accidents. Intelligent fault diagnosis technology formed by combining fault diagnosis and computer technology provides a useful reference for fault detection and equipment maintenance. Therefore, it is vital to apply intelligent fault diagnosis method to rolling bearings. Intelligent fault diagnosis method has been widely used in bearing fault diagnosis. Xu et al. [1] proposed a new expert system based on belief rules (BRB) built from multiple activated BRB subsystems in the meantime for diagnosing 
whether the marine diesel engines were faulty. Pang et al. [2] proposed a novel fault pattern classification method based on an ensemble kernel extreme learning machine (KELM) that fuses features of time and frequency domains into intrinsic features that are low dimensional using local and global principal component analysis. Li et al. [3] proposed a novel machine fault diagnosis method that can efficiently learn discriminative representations with input data's local and global geometry. Kaplan et al. [4] proposed a new approach based on texture analysis that converts vibration signals to grayscale images to fuse non-whole binary patterns and texture features for bearings fault diagnosis. Deng et al. [5] proposed a modified classification and regression tree (CART) algorithm to improve fault diagnosis speed by decreasing the numbers of iteration in computation to guarantee accuracy. Zhao et al. [6] proposed a new deep residual network based on multiple wavelet coefficients fusion for fault diagnosis. Ma et al. [7] proposed a new fault detection and diagnosis (FDD) method that built an overcomplete dictionary pair based on a dictionary pair learning strategy from features extracted from the wavelet transform for motor fault diagnosis. Li et al. [8] suggested a novel feature extraction method that can combine learnable modules of multiply LS-SVMs in the structure of deep stacking based on representation learning (S-RL) to extract features for fault diagnosis. Zheng et al. [9] proposed an improved MPE-based feature extraction method to extract the fault features from the vibration signal of rolling bearing. They applied the PSO-based SVM to fault diagnosis. Deng et al. [10] proposed an optimized deep belief network model based on an improved quantum-inspired differential evolution algorithm for realizing the fault diagnosis of rolling bearings. Zhao et al. [11] proposed a new high-order differential mathematical morphology gradient spectrum entropy method to extract rolling bearing's vibration signal features. Zhao et al. [12] proposed a novel method that applied principal component analysis and broad learning system to fault diagnosis. Deng et al. [13] proposed a novel intelligent diagnosis method based on LS-SVM with enhanced PSO algorithm for fault of rolling bearing.

Like other mainstream machine learning methods [14], such as SVM [15-25], fuzzy $k$-nearest neighbor method (FKNN) [26-30], and neural networks [31-33], KELM has been employed in many fields [34-40]. Du et al. [41] proposed a discriminative manifold ELM autoencoder to extract discriminative features from vibration measurements. The result achieved a $2 \%$ improvement in testing accuracy compared with the KELM. Wang et al. [42] developed a new ensemble ELM for compound-fault diagnosis of rotating machinery. Compared to the existing multilabel classifiers, the experimental results demonstrated that the proposed method achieved the best performance. Tian et al. [43] proposed an ensemble hierarchical ELM with deep learning architecture for unsupervised feature learning and supervised classification. The results showed that the proposed method performed better than traditional ELM and its variants. Zhong et al. [44] proposed a new fault diagnosis method based on a sparse Bayesian ELM. The proposed method could identify the single and simultaneous fault more quickly and precisely when compared against traditional techniques.

The KELM is one of the ELMs which is constructed based on kernel tricks. The capability of KELM is mainly affected by two critical parameters: one is the penalty coefficient, and the other is the kernel width. At present, researchers have proposed many effective methods to determine two critical parameters in KELM. Lu et al. [45] proposed to use PSO to optimize the parameters of KELM for obtaining the optimal model. Luo et al. [46] developed a multistrategy improved GOA-based KELM for bankruptcy prediction. Wang et al. [47] planned to use a chaotic FOA optimized KELM to diagnose sepsis. Tian et al. [48] utilized the quantum-based PSO optimized KELM for activity recognition. Baliarsingh et al. [49] offered a weighted-chaotic SSA for simultaneously optimizing KELM parameters and features in the genomic data. Hu et al. [50] developed cross-validated PSO for training an optimal KELM for fault diagnosis of wind turbine gearbox. Pani and Nayak [51] suggested employing KELM based on the chaotic GSA to prognose the solar irradiance. Luo et al. [52] recommended using GWO-MFO to achieve the optimal KELM model for diagnosing somatization disorder. Li et al. [53] offered a novel method that uses the improved binary GWO wrapped with KELM for disease diagnosis. Bisoi et al. [54] proposed using DE to train an optimal KELM to predict stock price and movement. Wang et al. [55] proposed a chaotic MFO used to optimize the critical parameters of KELM to obtain an optimal KELM model for medical diagnosis. Wang et al. [56] proposed obtaining an optimal KELM model by using GWO to predict the bankruptcy of the enterprise. Heidari et al. [57] proposed an improved GWO based on a multistrategy enhanced using effective exploratory and exploitative mechanisms. Chen et al. [58] proposed using chaotic and mutative BFO to seek the optimal parameters of KELM for classification tasks.

In this study, an improved butterfly optimization algorithm- (BOA-) optimized KELM model (SBOA-KELM) was proposed and applied to bearing fault diagnosis. First, the energy entropy features are extracted from the raw vibration signals by CEEMDAN. The original vibration signals were decomposed into multiple IMF components by CEEMDAN. The energy entropy of the IMFs was calculated to construct an energy feature vector. Second, to avoid data redundancy caused by smaller energy features and increase calculation, a random forest was used to evaluate feature's importance and select informative features as new feature vectors. Third, the proposed SBOA-KELM method was used for fault feature classification. Finally, the proposed SBOAKELM was verified and compared with several representative approaches. The experimental results presented that the proposed technique effectively diagnosed the bearing faults. The average classification accuracy was much improved. Table 1 lists the nomenclatures in the paper.

The whole structure of the study is structured as follows. Section 2 explains the data collection and gives a brief description of the data collection, CEEMDAN, random forest, SBOA, and proposed SBOA-KELM model. The experimental setup is termed in Section 3. Section 4 explains the 
TABLE 1: Nomenclatures in the paper.

\begin{tabular}{lc}
\hline Nomenclature & \\
\hline BOA & Butterfly optimizer algorithm \\
KELM & Kernel extreme learning machine \\
IMFs & Intrinsic mode functions \\
SSA & Salp swarm algorithm \\
FDD & Fault detection and diagnosis \\
PSO & Particle swarm optimization \\
GWO & Grey wolf optimizer \\
MFO & Moth-flame optimization \\
BFO & Bacterial foraging optimizer \\
CPSO & Cross-validated particle swarm optimizer \\
ITD & Intrinsic timescale decomposition \\
CBA & Cloud bat algorithm \\
CV & Cross-validation \\
CEEMDAN & Complete ensemble empirical mode decomposition based on adaptive noise \\
BA & Bat-inspired algorithm \\
SCA & Sine cosine algorithm \\
WOA & Whale optimization algorithm \\
SCADE & Cauchy and Gaussian SCA \\
CGSCA & A-C parametric WOA \\
ACWOA & Chaotic WOA \\
CWOA & Sine cosine algorithm with differential evolution \\
DM-ELM-AE & Pairwise-coupled sparse Bayesian extreme learning machine \\
EELM & Pairwise-coupled probabilistic neural networks \\
PC-SBELM & Pairwise-coupled relevance vector machine \\
PC-PNN & Ensemble manifold ELM autoencode \\
PC-RVM & Discriminative morion
\end{tabular}

results of SBOA on benchmark functions and SBOA-KELM on the bearing dataset. The conclusions and future works are delivered in Section 5.

\section{Materials and Methods}

2.1. Data Collection. The rolling bearing data on the website of the Bearing Data Center of Case Western Reserve University were employed in this study to check the feasibility and utility of the proposed method. The URL of the website of the Bearing Data Center is https://csegroups.case.edu/ bearingdatacenter/home.

The rolling bearing model is 6205-SKF. The structural parameters of rolling bearings are shown in Table 2. First, damage points are artificially set on the inner race, outer race, and ball of rolling bearings. Under the condition of input shaft speed $n=1797 \mathrm{r} / \mathrm{min}$ and the acquisition frequency of $12 \mathrm{kHz}$, the bearing's vibration signals in four states, normal state, inner race fault, outer race fault, and ball fault, are gathered. There are 40 sets of sample data gathered from the vibration signals of each state. The length of each sample data is 1200 .

2.2. SBOA-KELM Method. The flowchart of the proposed SBOA-KLEM is shown in Figure 1. The whole flow includes feature extraction based on CEEMDAN energy entropy, feature selection based on random forest, and classification based on BOA-KELM. The first step is to extract features, using the CEEMDAN method to decompose the raw vibration signals of bearing into multiple IMFs, computing each IMF's energy entropy and normalizing. The second step is to select the feature from the CEEMDAN energy entropy to reduce data redundancy. The third step is to optimize the two critical parameters of the KLEM using SBOA. Then, the optimal parameters and feature combination are used to train an optimal KELM. Finally, the optimal KELM classifier is diagnosed with the rolling bearing to determine the bearing's working condition. The standard 10-fold cross model is used to divide the data to obtain a more exact and unbiased experimental result, which many researchers often adopt.

2.2.1. Feature Extraction. Fault feature extraction is a critical step in the fault diagnosis of rolling bearings [59]. When rolling bearings are in abnormal faults, vibration signals are mostly nonstationary and nonlinear characteristics, and they are interfered with intense noise [60].

After that, comparing with the effect of EEMD on feature extraction, the CEEMDAN has a more excellent performance to preserve the original signal and eliminate noise and extract bearing fault features more accurately and timely. The CEEMDAN is an adaptive time-frequency signal analysis method developed based on EEMD, which can effectively extract fault frequency characteristics. Based on previous research, CEEMDAN has a better effect on signal decomposition than EEMD [61].

It needs to be quantified [62] to make the fault characteristic information after CEEMDAN decomposition more apparent. When different faults occur in rolling bearings, the amplitude energy within the vibration signal's 
TABLE 2: The structural parameters of rolling bearings.

\begin{tabular}{lccccc}
\hline $\begin{array}{l}\text { Outer ring diameter } \\
(\mathrm{mm})\end{array}$ & $\begin{array}{c}\text { Inner ring diameter } \\
(\mathrm{mm})\end{array}$ & $\begin{array}{c}\text { Bearing pitch diameter } \\
(\mathrm{mm})\end{array}$ & $\begin{array}{c}\text { Ball diameter } \\
(\mathrm{mm})\end{array}$ & $\begin{array}{c}\text { Number of } \\
\text { balls }\end{array}$ & $\begin{array}{c}\text { Contact angle } \\
\left({ }^{\circ}\right)\end{array}$ \\
\hline 51.99 & 25.00 & 31.04 & 7.94 & 9 & 9 \\
\hline
\end{tabular}

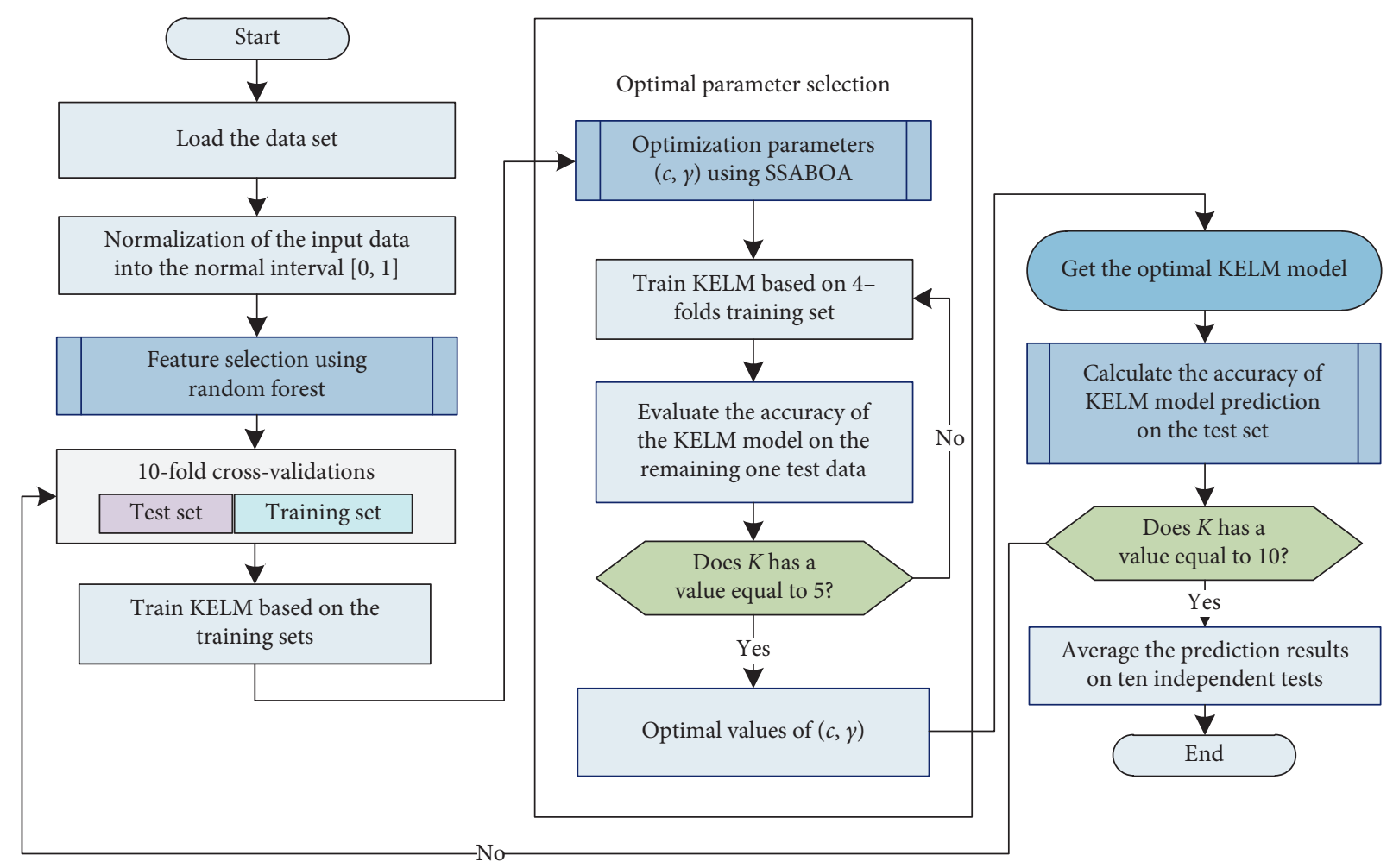

Figure 1: Flowchart of SBOA-KELM.

frequency range will change to varying degrees. Therefore, the feature matrixes of bearing faults consist of the energy entropy of the IMFs. The method of feature extraction based on CEEMDAN energy is as follows:

Step 1: CEEMDAN will decompose the vibration fault signal of the rolling bearing to obtain multiple IMFs:

$$
x(t)=\sum_{j=1}^{n} c_{j}(t)+r(t)
$$

Step 2: calculate the amplitude energy $E_{1}, E_{2}, \ldots, E_{n}$ of each IMF component:

$$
E_{j}=\sum_{k=1}^{N}\left|c_{j}(k)\right|^{2}
$$

In equation (2), $N$ is the number of sampling points of the $j$-th IMF component.
Step 3: assuming that $r(t)$ can be ignored, the total energy of the signal obtained is

$$
E_{\text {sum }}=\sum_{j=1}^{n} E_{j}=\sum_{j=1}^{n} \sum_{k=1}^{N}\left|c_{j}(k)\right|^{2} .
$$

Step 4: in order to avoid that the IMF components in the partial amplitude energy concentration control the relatively weak IMFs, the amplitude energy of each order IMF is normalized:

$$
p_{j}=\frac{E_{j}}{E_{\text {sum }}} .
$$

Therefore, the corresponding CEEMDAN energy entropy $(\mathrm{EN})$ can be denoted as

$$
H_{\mathrm{EN}}=-\sum_{j=1}^{n} p_{j} \log p_{j} .
$$


In equation (5), $p_{j}$ is the proportion of the $j$-th IMF amplitude component in the total energy.

\subsubsection{Feature Selection Based on Random Forest.} Random forest is a multiclassifier integrated algorithm that can obtain higher classification accuracy in a short time with fewer training samples [63]. For multitime domain feature sets, random forest classifiers can reduce dimensionality for features and reduce overfitting. The algorithm flow is as follows:

Step 1: the sample data's energy entropy features are input into the random forest to calculate feature importance and sort features in the descending order of feature importance

Step 2: according to a certain deletion ratio, delete from the feature set to construct a new feature set

Step 3: input the new feature set into a new random forest and calculate the importance of each feature, and then sort in the descending order and repeat Steps 2 and 3 until a specified number of features are left

Step 4: each feature set corresponds to a random forest; calculate the corresponding out-of-bag error rate, and take the feature set with the lowest out-of-bag error rate as the last selected feature set

2.2.3. Classification Based on BOA-KELM. Optimization can be formulated in many ways, including multiobjective, fuzzy variables, larger-scale, or robust optimization. One way to deal with a problem is to have a single objective and hybrid methods for solving it [64-72]. In this study, an SBOA-KELM was constructed by improving BOA by introducing the SSA. The resultant SBOA was taken to handle the problem of parameter optimization of KELM for the fault classification task. BOA [73] was a novel nature-inspired optimization algorithm based on the food foraging of butterflies. Butterflies are the main object of BOA's search and work efficiently to complete the optimized search of the best working solution in space [74].

In this study, KELM was to identify the fault types of rolling bearings. In KELM, the output results are determined by calculating the kernel function without the hidden-layer output matrix. Compared with SVM, KELM performs faster learning speed and has better generalization performance [75]. Therefore, KELM was chosen to diagnose the faults of rolling bearings in this study.

For the past few years, many scientific researchers were continually exploring the application of KELM in fault diagnosis of rotating machinery. $\mathrm{Hu}$ et al. [50] proposed a fault diagnosis method that extracted time-domain features from vibration signal by using a wavelet packet transform (WPT) filter and diagnosed gearbox-related faults using a cross-validated particle swarm optimized- (CPSO-) based KELM. Lei et al. [76] proposed a new fault classification method that combined KELM with the intrinsic timescale decomposition (ITD) technique to identify the tool wear conditions. Long et al. [77] proposed a novel fault patterns methodology of wind turbine gearbox that combines a cloud bat algorithm (CBA) with KELM. Wang et al. [78] proposed a novel bearing intelligent fault diagnosis method that optimized KELM parameters through the krill herd algorithm (NKH). In this study, the two critical parameters of KELM are optimized by SBOA to improve the classification accuracy of fault diagnosis. The flowchart of SBOA is shown in Figure 2.

2.2.4. Proposed SBOA-KELM. After normalizing the CEEMDAN energy entropy features, the random forest was established to obtain the optimal feature subset that reduces data redundancy. To promote the classification accuracy of fault identification and the model's generalization ability, the SBOA model is proposed to obtain the optimal key parameters value of KELM. The steps of feature selection based on random forest and parameter optimization of the SBOAKELM model are as follows:

Step 1: normalize the energy entropy feature data, and the range of normalized data is $[0,1]$.

Step 2: the importance of the normalized features is evaluated by random forest through out-of-band errors, and after setting the threshold, the optimal feature subset is selected.

Step 3: the optimal feature subset is separated into the training set and test set by 10 -fold cross-validation (CV) scheme.

Step 4: SBOA and KELM optimize the two critical parameters of KELM that is trained on the training set by inputting with the optimal feature subset through the inner 5-fold CV scheme.

Step 5: evaluate the accuracy of KELM on the test data. If the value of $K$ is less than 10, go to Step 4 .

Step 6: average the prediction result on ten independent tests as the output result.

\section{Experimental Setup}

The vibration signal was decomposed by CEEMDAN using the pyEMD toolkit in Python. The development tool uses PyCharm. After extracting the energy entropy feature from the IMF component decomposed by the vibration signal, it is saved as a CSV file to prepare for the next feature extraction and state recognition. The methods mentioned in this article, including SBOA and KELM, were implemented using MATLAB.

Data were scaled between 0 and 1 before extracting features. To make sure of fair results, classification accuracy is evaluated by the stratified 10 -fold CV. It means that the data were segmented into ten parts, of which five were used as training datasets of the SBOA-KLEM model and the remaining one as the test dataset. The entire flow was rotated ten times, with the average of the 10 test data being the final result. The number of the maximum iterations and swarm size were set at 50 and 20 , respectively. The seeking range for the two critical parameters in KELM is set as follows: $C \epsilon$ $\left[2^{-5}, 2^{5}\right], \gamma \in\left[2^{-5}, 2^{5}\right]$. 


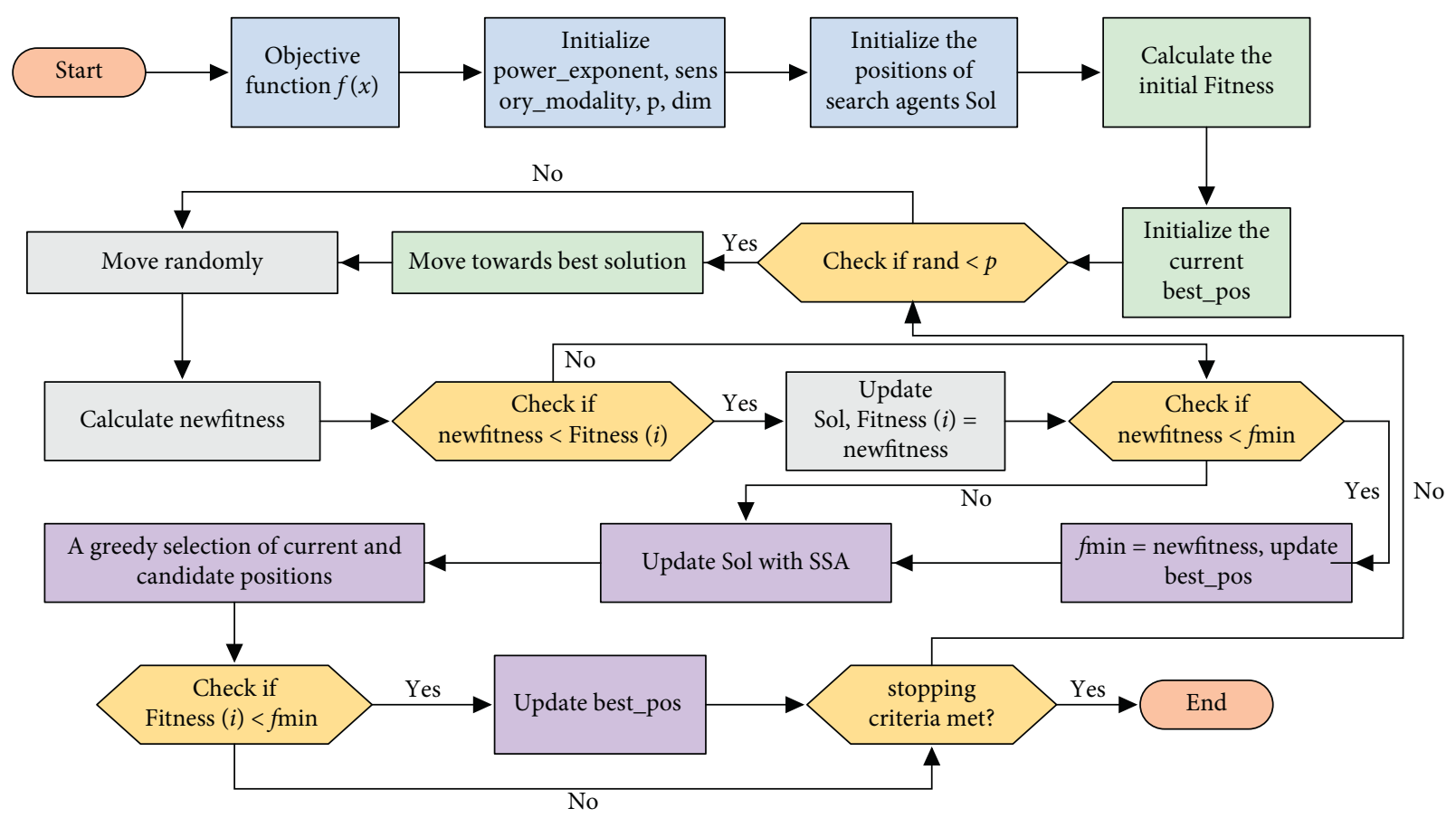

FIGURE 2: Flowchart of SBOA.

To evaluate the validity of the SBOA-KELM model, classification accuracy was analyzed and verified by the $10-$ fold CV procedure.

\section{Experimental Result}

4.1. Benchmark Function Validation. Some representative algorithms have been selected in this paper, including the classic original algorithms BA [79], SCA [80], WOA [81, 82], and BOA, and some improved algorithms including SCA_PSO [83], SCADE [84], CGSCA [85], CBA [86], ACWOA [87], and CWOA [88]. Table 3 shows the specific parameter values of the involved algorithms. According to fair-minded comparisons in neural networks literature, the above algorithms are all executed and tested in MATLAB software under the same computing environments [89-91]. This can ensure there is no preference en route for a method because of a pro in its testing plan [92-96]. We have used the IEEE CEC2017 benchmark functions as a test function. In the experiment, the number of particles was set to 30 , the size was set to 30 , and the maximum number of evaluations was set to 300,000 . Each algorithm was performed independently 30 times to take the average.

To verify and test the capability of BOA, 30 different benchmark test functions were simulated. These benchmarking features identify various features of the algorithm, such as rapid convergence, speed of convergence, ability to step outside of the partial optimization, and ability to avoid premature convergence $[97,98]$. Table 4 details the test results of the SBOA algorithm and the comparison algorithm on the benchmark function, presenting the average adaptation value and standard deviation of the algorithm run independently 30 times on the benchmark function,
TABLe 3: Parameters of the involved methods.

\begin{tabular}{lc}
\hline Method & Parameter values \\
\hline SBOA & $p=0.8 ;$ power_exponent $=0.1 ;$ \\
sensory_modality $=0.01$
\end{tabular}

respectively. The overall effect of the proposed SBOA is better than its other counterparts. The Friedman test [99] was used to test the algorithm performance. This test is based on the algorithm's strengths and weaknesses. The table has shown that SBOA has an ARV value of 1.6, superior to all the other competitive algorithms. The statistical results of each optimization task indicate that SBOA has a faster convergence rate.

Figure 3 shows the convergence trend of the algorithm over nine benchmark functions. It is possible to know from the figure that the convergence trend of this paper's algorithm on these nine benchmark functions is superior to other comparative algorithms. In the convergence trend diagram, this paper's improved convergence trend is significantly better in the middle of convergence. The experimental results demonstrate the effectiveness of SSA in BOA. In each test case, the final output of the SBOA best meets the 


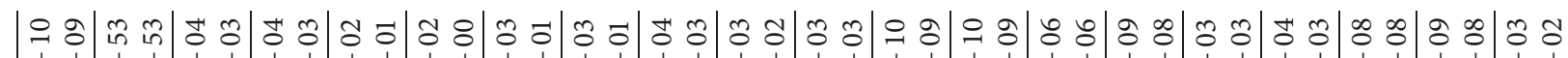

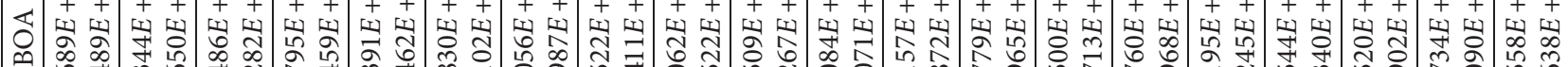

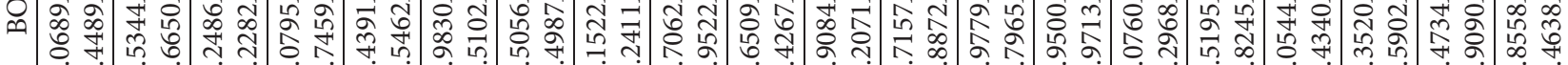

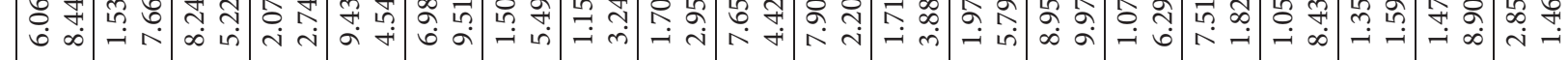

\&

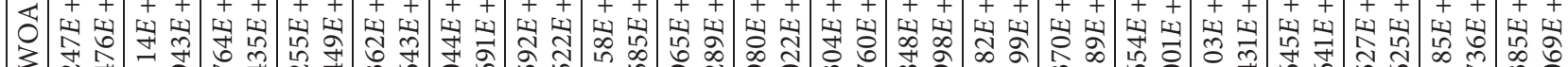

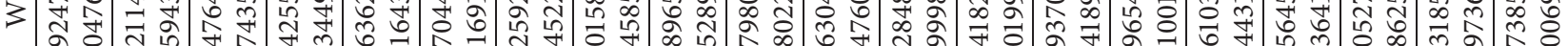

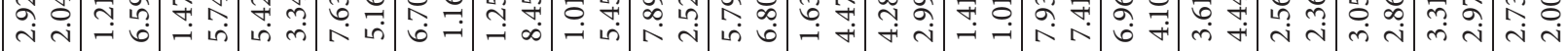
응

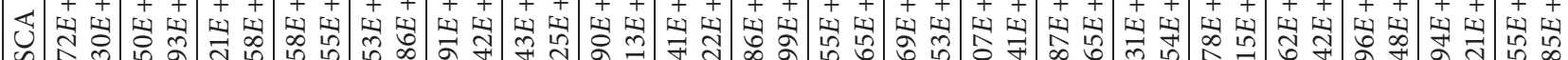

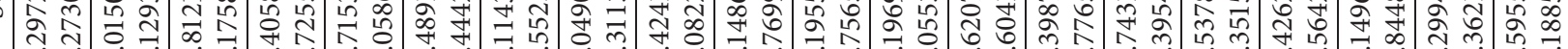

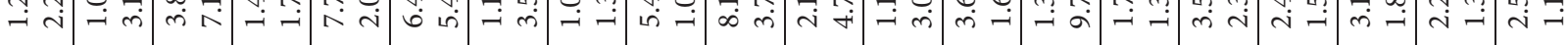

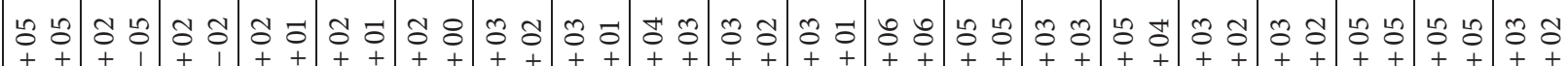

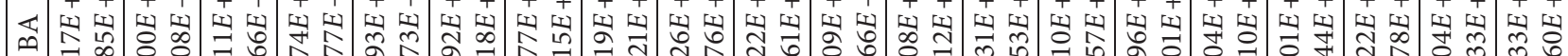
तิ

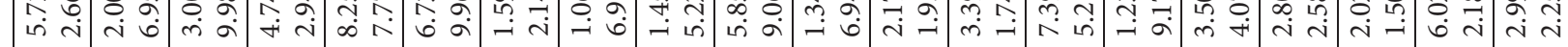

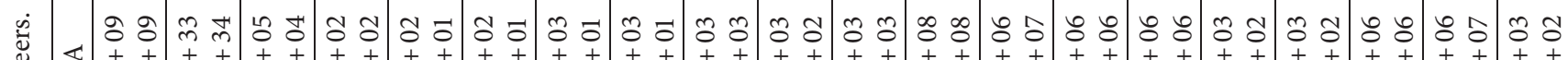

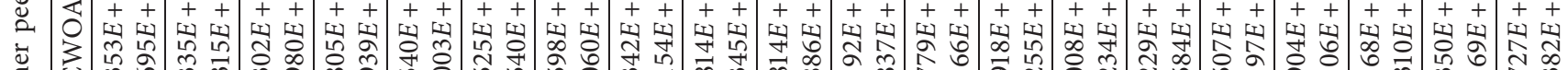

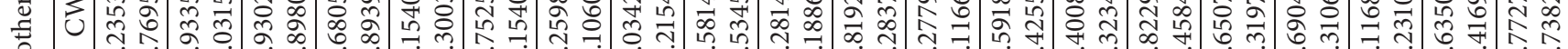
สี 4 $\rightarrow$ i

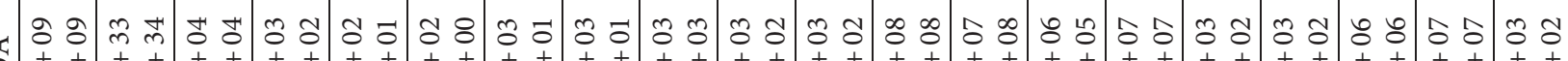
क ल) 㟧

ช

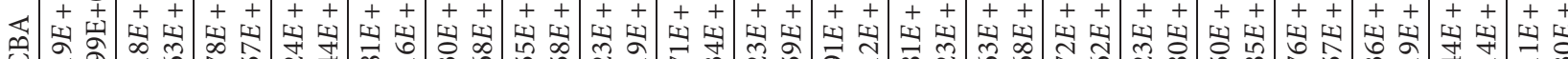

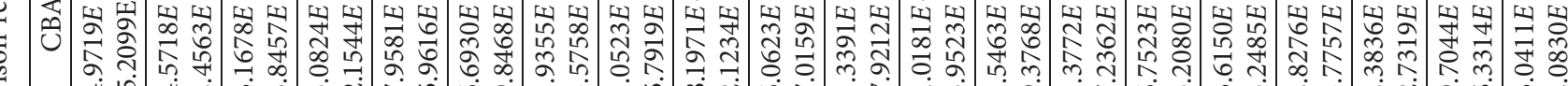
节

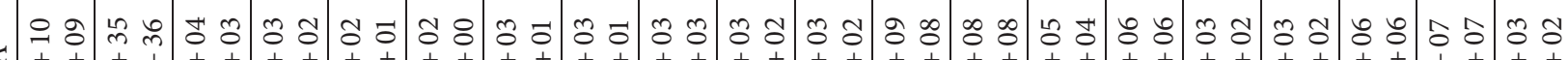

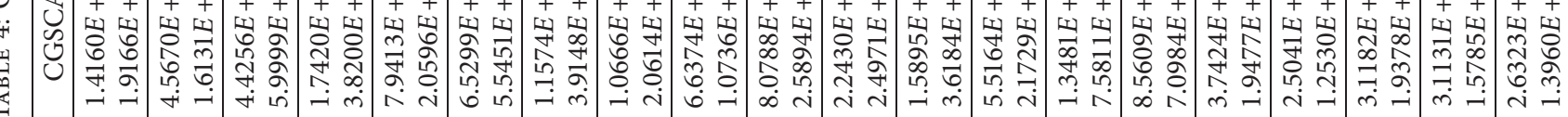

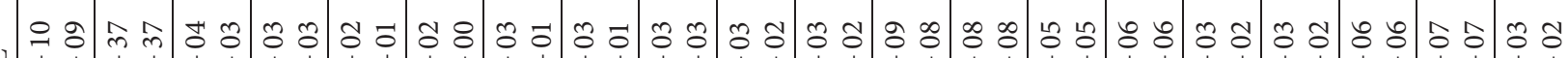

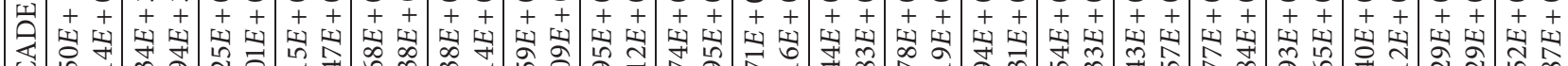

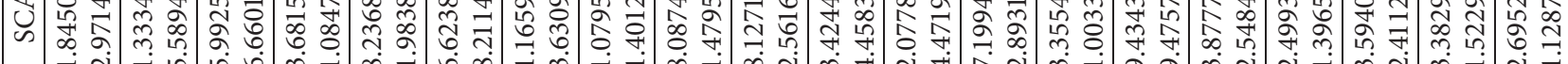

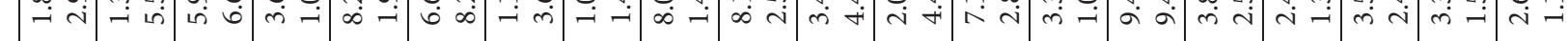

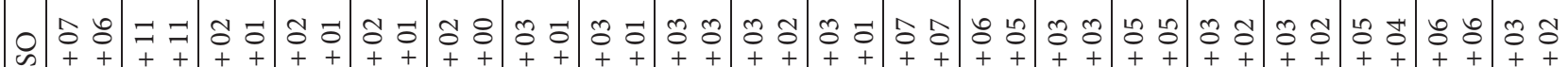

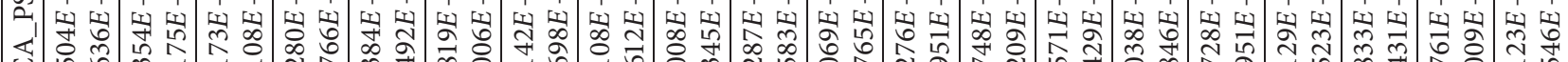

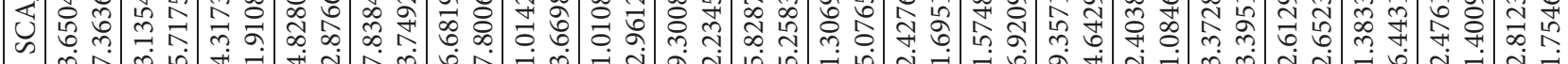
ช

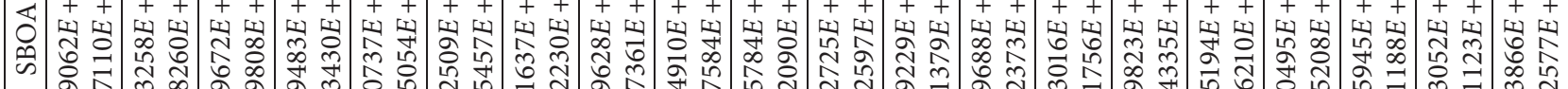

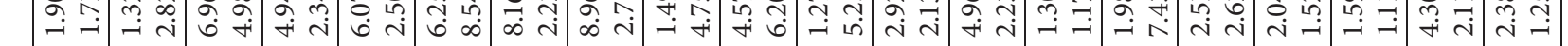

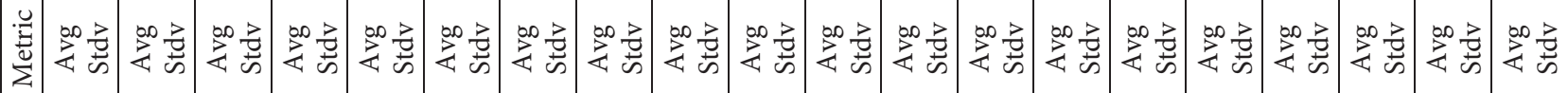

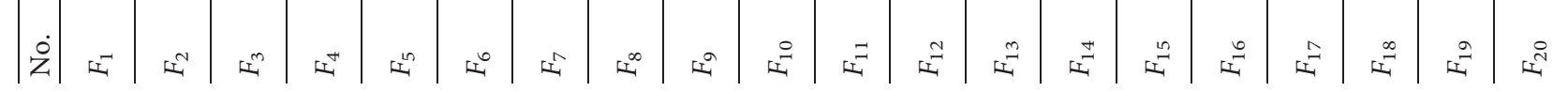




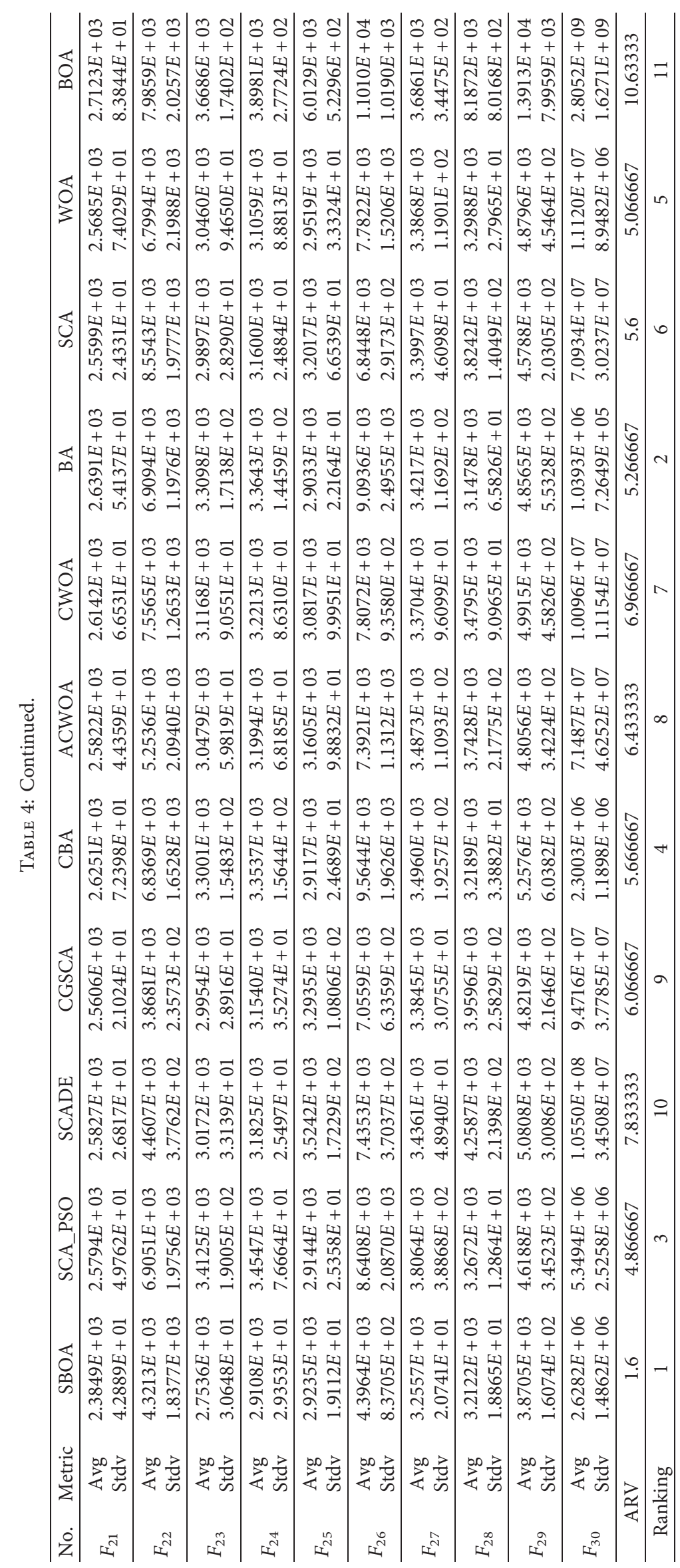



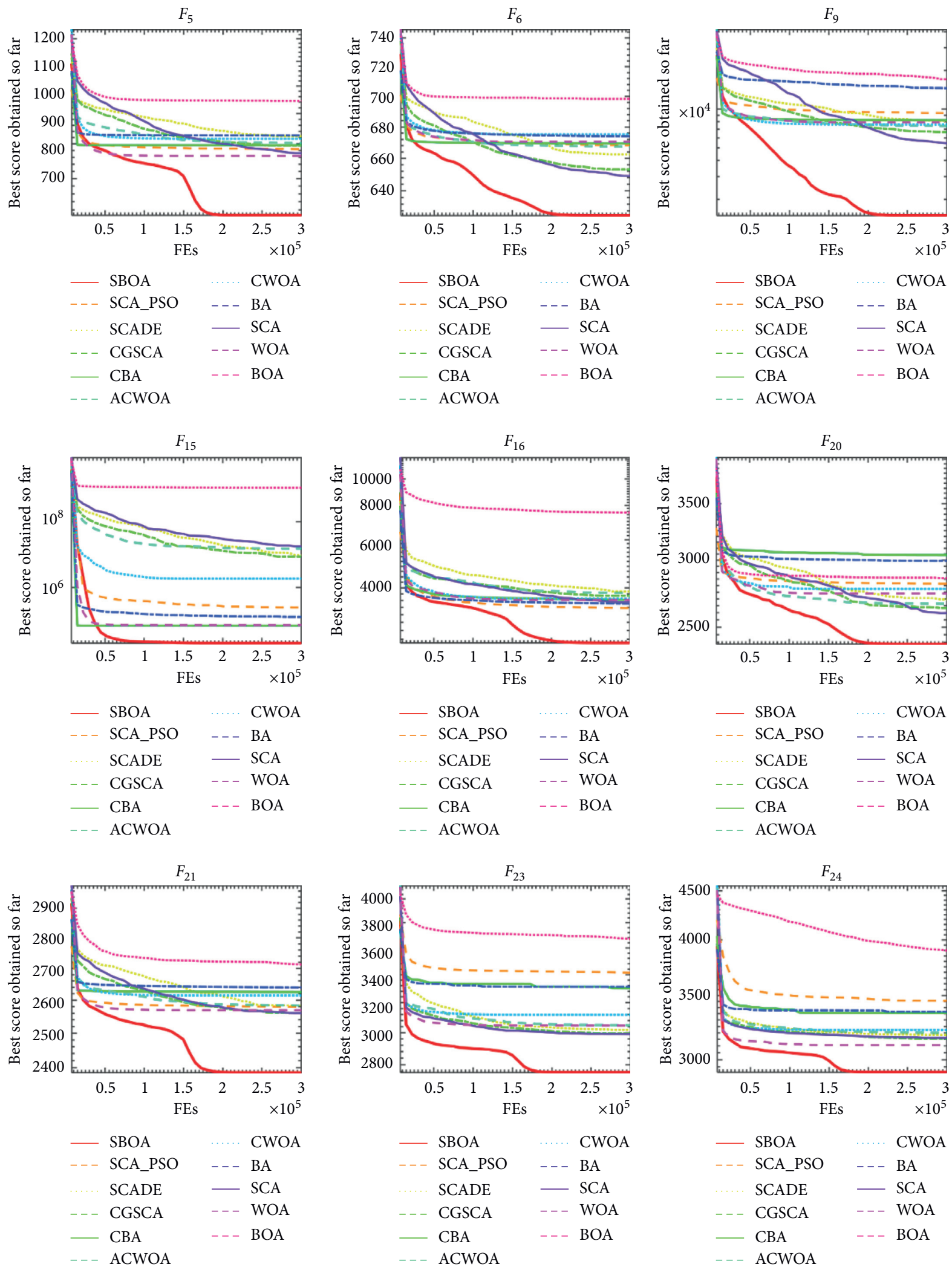

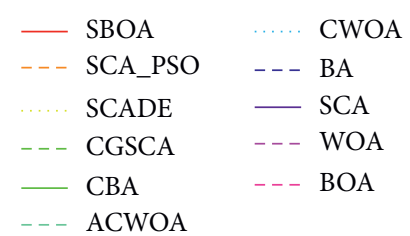

(a)

Figure 3: Continued. 

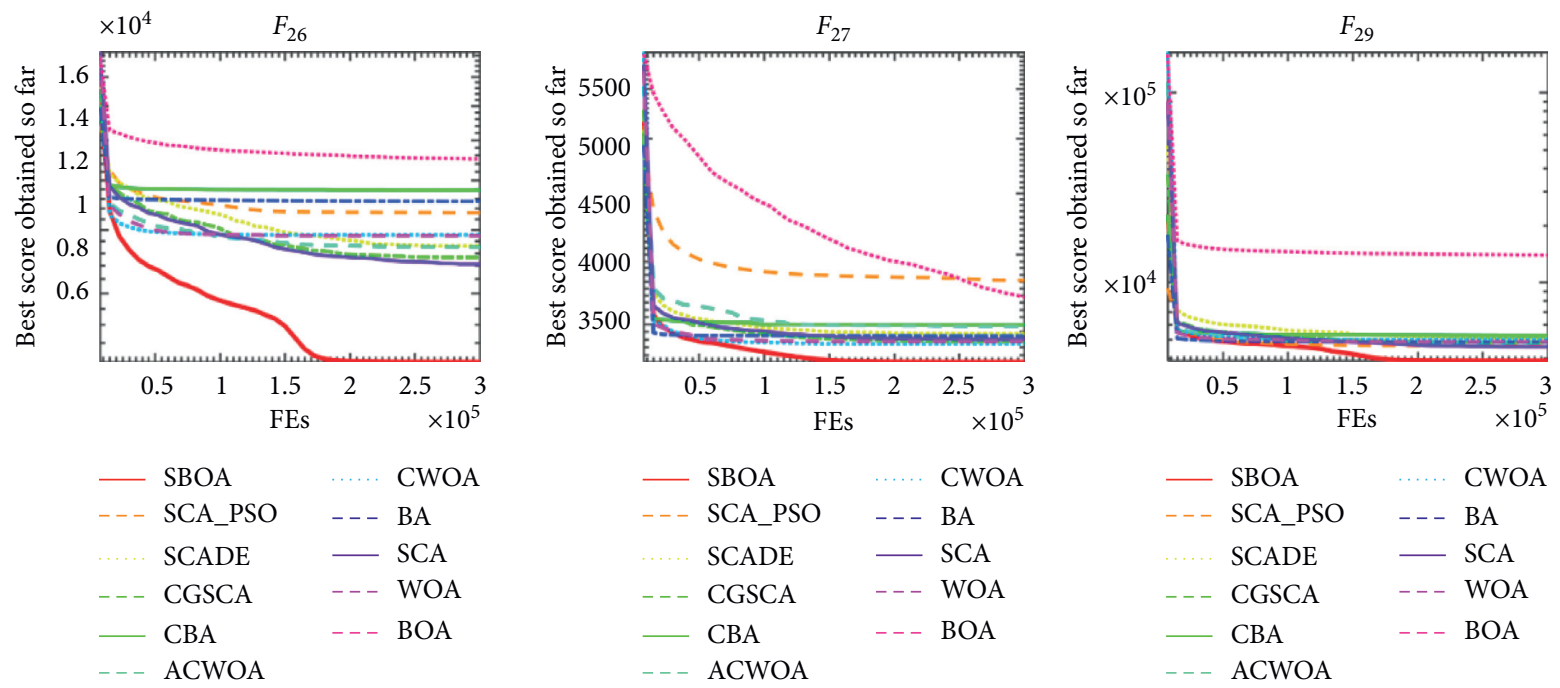

(b)

FIgURE 3: Convergence curves of 9 selected benchmark functions.

functional desires. For most test functions, SBOA obtains the best minimum value before arriving at the maximum number of iterations. Finally, the full results confirm the superiority of SBOA in terms of statistical results, reliability of global optimality, and algorithm success rate.

4.2. Prediction Results of Fault Diagnosis. In this paper, a series of IMFs are obtained by decomposing the vibration signals using CEEMDAN in the four rolling bearings states. For reasons of space, only the IMFs of the ball failure signal are listed, as shown in Figure 4. The time-domain features of the $\mathrm{IMF}_{1}-\mathrm{IMF}_{6}$ components are more prominent and contain most of the feature information. To further illustrate the advantages of CEEMDAN in vibration signal analysis, the signal completeness, orthogonality, and time consumption after CEEMDAN decomposition are calculated for the four states. The results are shown in Table 5. It shows that CEEMDAN is better than EEMD in orthogonality and completeness, although slightly larger in time, but negligible for modern computers.

To illustrate that the CEEMDAN energy entropy can reflect the four states of the roller bearing, the CEEEMDAN energy entropy values are calculated for the different states of the roller bearing. As shown in Table 6, the results show that the CEEEMDAN energy entropy values of bearings in different states are different. The timescale of the IMF components is relatively average when the bearings are in the normal state. Hence, the CEEEMDAN energy entropy value of bearings in the normal state is the largest. The other three states of the failure's energy entropy are not the same because the amplitude energy changes to a different degree at different parts of the failure.
A random forest is constructed to perform feature selection on energy entropy feature vectors to remove redundant features. The random forest is constructed to evaluate the contribution of the seven energy entropy features for discriminating the fault status. The result is shown in Figure 5.

The features were arranged in ascending order of contribution with feature numbers $E_{7}, E_{6}, E_{3}, E_{5}, E_{2}, E_{1}$, and $E_{4}$. At a time, one feature was removed from the feature set to form a feature subset; thus, a subset of seven features is constructed. A subset of these features is input to the SBOAKELM model for fault identification and calculates the classification accuracy.

In this experiment, the SBOA-KELM model's validity is evaluated, and detailed results are shown in Table 7 . The average accuracy of classification obtained by SBOA-KELM is $100 \%$ in the table. Moreover, we observed that the SBOA had achieved the optimal parameters of KELM on the optimal feature space obtained from the random forest, which indicates that the SBOA has a good optimization capability for searching the optimal values. To guarantee this paper's proposed technique's validity, we compared SBOA-KELM with KELM and BOA-KELM models. The results are shown in Figure 6. As shown, the classification accuracy of SBOAKELM is better than both KELM and BOA-KELM, where the average classification accuracy of KELM is $98.12 \%$, and BOA-KELM is $99.38 \%$. The classification accuracy of SBOAKELM has reached $100 \%$, which is $1.88 \%$ better than the average accuracy of KELM and $0.62 \%$ better than the average accuracy of BOA-KELM, overcoming the underlearning problem on a small sample and has shown more robust generalization performance. 

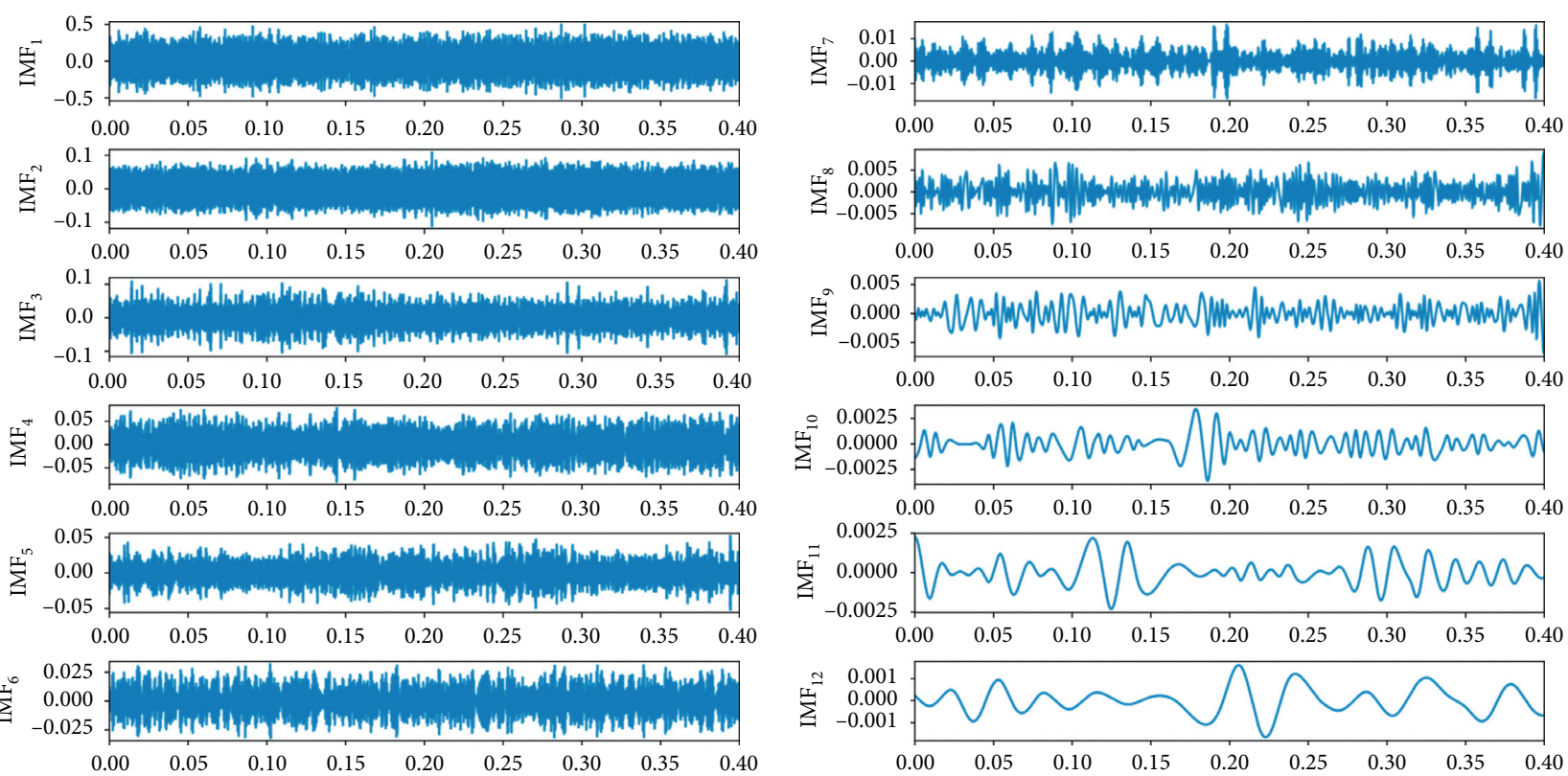

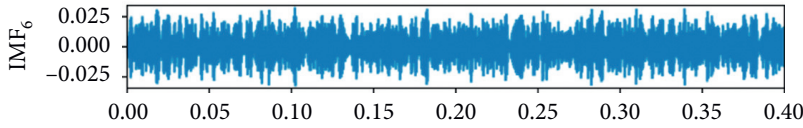

FIgURE 4: Time-domain diagram of the IMF components of a vibration signal based on a rolling ball fault.

TAble 5: Performance comparison of CEEMDAN and EEMD.

\begin{tabular}{|c|c|c|c|c|}
\hline Experiment type & Status of the rolling bearing & Orthogonality & Completeness & Time $t(\mathrm{~s})$ \\
\hline \multirow{4}{*}{ EEMD } & Normal & 0.1840 & $2.5469 e-07$ & 12.11 \\
\hline & Inner race failure & 0.0366 & $2.5469 e-07$ & 13.29 \\
\hline & Ball failure & 0.0155 & $2.5469 e-07$ & 13.12 \\
\hline & Outer race failure & 0.0128 & $2.5469 e-07$ & 13.43 \\
\hline \multirow{4}{*}{ CEEMDAN } & Normal & 0.1389 & $2.5469 e-34$ & 17.68 \\
\hline & Inner race failure & 0.0246 & $4.3318 e-33$ & 17.82 \\
\hline & Ball failure & 0.0081 & $2.3366 e-32$ & 19.32 \\
\hline & Outer race failure & 0.0063 & $8.8566 e-34$ & 19.52 \\
\hline
\end{tabular}

TABLE 6: Entropy value of four states of the rolling bearing.

\begin{tabular}{lcccc}
\hline Status of the rolling bearing & Normal & Inner race failure & Ball failure & Outer race failure \\
\hline Entropy & 0.7545 & 0.4331 & 0.5554 & 0.6371 \\
\hline
\end{tabular}

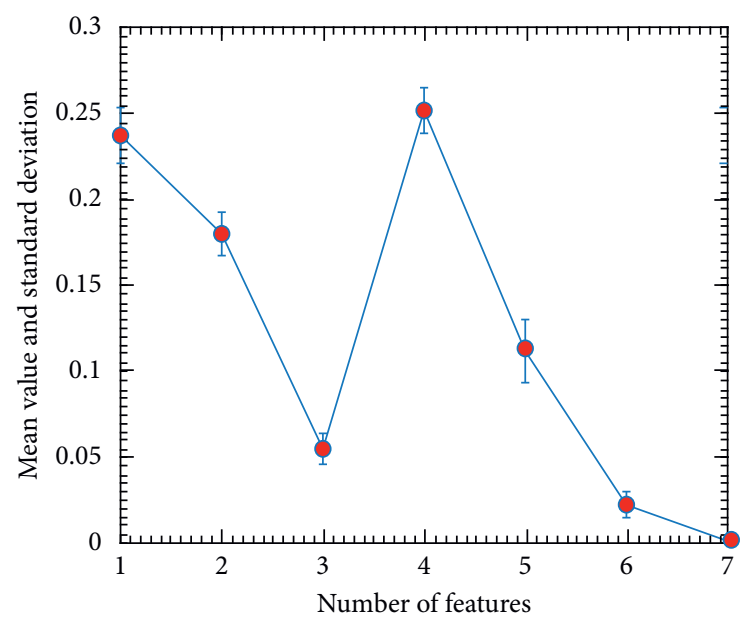

Figure 5: The degree of importance of the energy entropy features. 
TABLE 7: Feature selection based on the random forest via the 10 -fold CV procedure.

\begin{tabular}{lccccccc}
\hline Fold & $\left\{E_{4}\right\}$ & $\left\{E_{1}, E_{4}\right\}$ & $\left\{E_{2}, E_{1}, E_{4}\right\}$ & $\left\{E_{5}, E_{2}, E_{1}, E_{4}\right\}$ & $\left\{E_{3}, E_{5}, E_{2}, E_{1}, E_{4}\right\}$ & $\left\{E_{6}, E_{3}, E_{5}, E_{2}, E_{1}, E_{4}\right\}$ & $\left\{E_{7}, E_{6}, E_{3}, E_{5}, E_{2}, E_{1}, E_{4}\right\}$ \\
\hline No. 1 & 0.6880 & 0.9380 & 1.0000 & 1.0000 & 0.938 & 1.0000 & 1.0000 \\
No. 2 & 0.8750 & 1.0000 & 1.0000 & 1.0000 & 1.0000 & 1.0000 & 1.0000 \\
No. 3 & 0.7500 & 1.0000 & 1.0000 & 1.0000 & 1.0000 & 1.0000 & 1.0000 \\
No. 4 & 0.9380 & 1.0000 & 1.0000 & 1.0000 & 1.0000 & 1.0000 & 1.0000 \\
No. 5 & 0.8130 & 1.0000 & 1.0000 & 1.0000 & 1.0000 & 1.0000 & 1.0000 \\
No. 6 & 0.8750 & 0.9380 & 1.0000 & 1.0000 & 1.0000 & 1.0000 & 1.0000 \\
No. 7 & 0.7500 & 0.9380 & 1.0000 & 1.0000 & 1.0000 & 1.0000 & 1.0000 \\
No. 8 & 0.9380 & 0.9380 & 1.0000 & 1.0000 & 1.0000 & 1.0000 & 1.0000 \\
No. 9 & 0.8130 & 0.9380 & 1.0000 & 1.0000 & 1.0000 & 0.6880 & 0.0000 \\
No. 10 & 0.8130 & 1.0000 & 1.0000 & 1.0000 & 0.9380 & 0.9690 & 0.9940 \\
Avg & 0.8250 & 0.9690 & 1.0000 & 1.0000 & 0.9880 & 0.0990 & 0.0200 \\
Std & 0.0820 & 0.0330 & 0.0000 & 0.0000 & 0.0260 & & \\
\hline
\end{tabular}

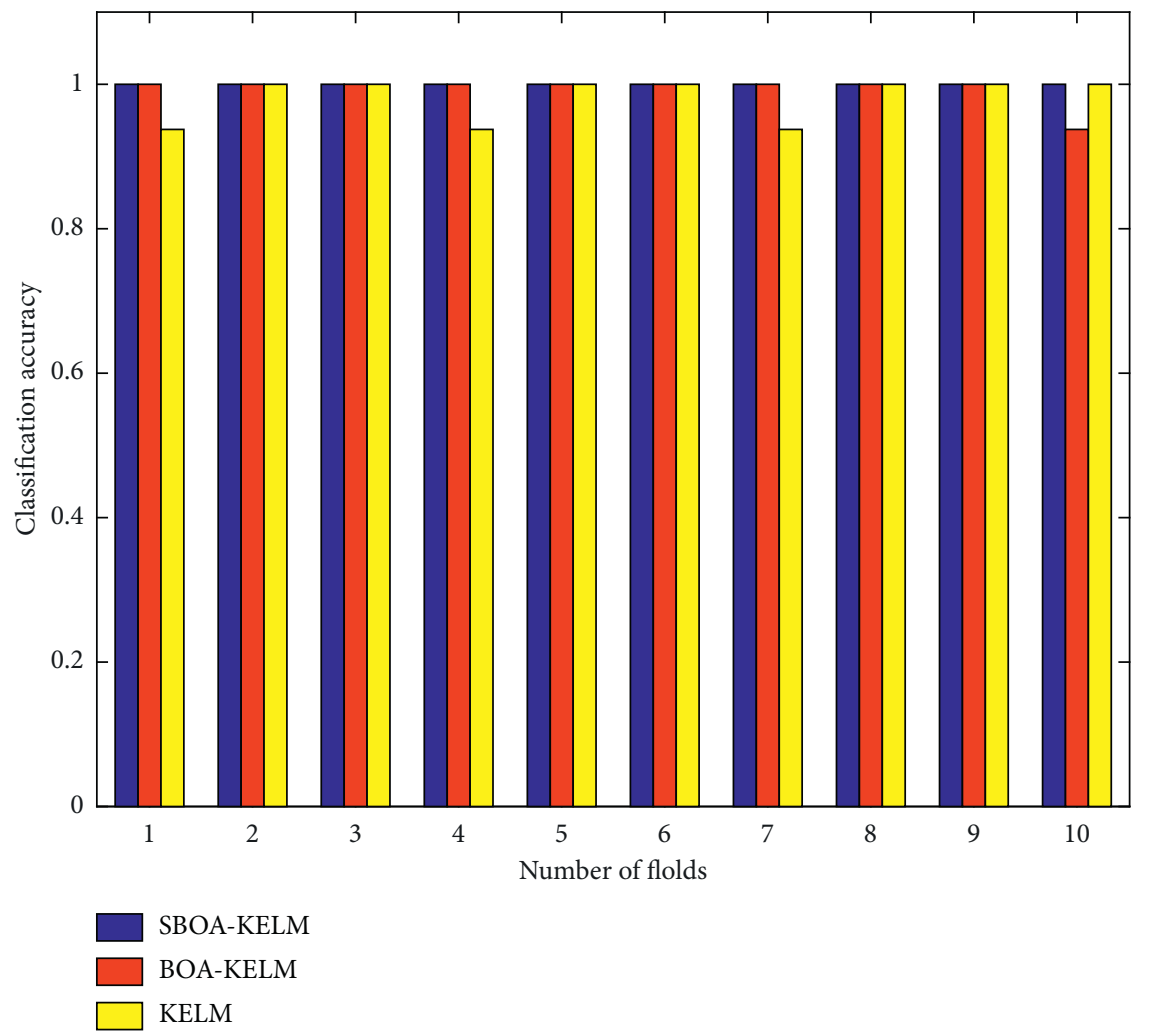

Figure 6: Performance comparison of SBOA-KELM, BOA-KELM, and ELM via the 10-fold CV procedure.

\section{Conclusions and Future Works}

In this study, an intelligent fault diagnosis model based on SBOA-KELM is established to identify the rolling bearings' running state. This approach's innovation introduces SSA into the $\mathrm{BOA}$ to get the right balance between the exploration and exploitation of BOA for the first time. Compared with ten other optimization algorithms, it was found that the presented method achieves better solution quality and smaller standard deviation on 30 IEEE CEC2017 benchmark problems. SBOA also showed the better capability to obtain a better combination of KELM parameters than the original BOA. The experimental results showed that the proposed
SBOA-KELM model had performed more accurately and stably than its counterparts in recognizing rolling bearings.

For future work, several aspects need to be further explored. The proposed SBOA-KELM method is intended to be used in the future for other aspects of rolling bearings, such as fault warning, real-time fault diagnosis, and live monitoring. The SBOA-KELM can also be combined with other feature extraction methods, such as high-order spectral analysis, inverse spectral analysis, wavelet transformation, and variable modal decomposition, further to enrich the fault diagnosis methods for rolling bearings. Moreover, the proposed method can be further applied to other scenarios including differentiation of malignant and 
benign thyroid nodules [100], diagnosis of Parkinson's disease [26-28, 36, 101], diagnosis or prognosis of paraquat-poisoned patients $[22,23,102,103]$, identification of poisoning status [104, 105], RNA secondary structure prediction [106], prediction optimization of cervical hyperextension injury [107], diagnosis of erythematosquamous diseases [108], other medical diagnosis problems [15, 25, 53, 55, 109-111], decision-making methods [112-114], parameter optimization [115], deep learning [116-118], image segmentation [119, 120], image marbleization [121], image colorization [122, 123], image editing [124], bankruptcy prediction [40, 46, 56, 125], face recognition [126], neural network configuration [127], information fusion [128], social evolution modelling [129], text clustering [130], recognition of facial microexpressions [131], unsupervised band selection [132], and other problems $[18,30,34,35,52,133,134]$.

\section{Data Availability}

The data used to support the findings of this study are available from the corresponding author upon request.

\section{Conflicts of Interest}

The authors declare that there are no conflicts of interest regarding the publication of the article.

\section{Authors' Contributions}

Weibin Chen, Changcheng Huang, and Huiling Chen contributed equally to this work.

\section{Acknowledgments}

This research was supported by the National Natural Science Foundation of China (U19A2061 and U1809209), Science and Technology Development Project of Jilin Province (20190301024NY and 20200301047RQ), Jilin Provincial Industrial Innovation Special Fund Project (2018C039-3), the Zhejiang Provincial Natural Science Foundation of China (LY21F020001), and Wenzhou Science and Technology Bureau (ZG2020026). Thanks are due to Ali Asghar Heidari (https://aliasgharheidari.com) for checking the English language of the paper.

\section{References}

[1] X. Xu, X. Yan, C. Sheng, C. Yuan, D. Xu, and J. Yang, "A belief rule-based expert system for fault diagnosis of marine diesel engines," IEEE Transactions on Systems Man Cybernetics-Systems, vol. 50, no. 2, pp. 656-672, 2020.

[2] S. Pang, X. Yang, X. Zhang, and X. Lin, "Fault diagnosis of rotating machinery with ensemble kernel extreme learning machine based on fused multi-domain features," ISA Transactions, vol. 98, pp. 320-337, 2020.

[3] Y. Li, C. K. L. Lekamalage, T. Liu, P.-A. Chen, and G.-B. Huang, "Learning representations with local and global geometries preserved for machine fault diagnosis," IEEE Transactions on Industrial Electronics, vol. 67, no. 3, pp. 2360-2370, 2020.
[4] K. Kaplan, Y. Kaya, M. Kuncan, M. R. Minaz, and H. Metin Ertunça, "An improved feature extraction method using texture analysis with LBP for bearing fault diagnosis," Applied Soft Computing, vol. 87, Article ID 106019, 2020.

[5] H. Deng, Y. Diao, W. Wu, J. Zhang, M. Ma, and X. Zhong, "A high-speed D-CART online fault diagnosis algorithm for rotor systems," Applied Intelligence, vol. 50, no. 1, pp. 29-41, 2020.

[6] M. Zhao, M. Kang, B. Tang, and M. Pecht, "Multiple wavelet coefficients fusion in deep residual networks for fault diagnosis," IEEE Transactions on Industrial Electronics, vol. 66, no. 6, pp. 4696-4706, 2019.

[7] D. Ma, Y. Lu, Y. Zhang, H. Bao, and X. Peng, "Bearing fault diagnosis based on collaborative representation using projection dictionary pair," Shock and Vibration, vol. 2019, Article ID 3871089, 13 pages, 2019.

[8] X. Li, Y. Yang, H. Pan, J. Cheng, and J. Cheng, "A novel deep stacking least squares support vector machine for rolling bearing fault diagnosis," Computers in Industry, vol. 110, pp. 36-47, 2019.

[9] J. Zheng, H. Pan, S. Yang, and J. Cheng, "Generalized composite multiscale permutation entropy and Laplacian score based rolling bearing fault diagnosis," Mechanical Systems and Signal Processing, vol. 99, pp. 229-243, 2018.

[10] W. Deng, H. Liu, J. Xu, H. Zhao, and Y. Song, "An improved quantum-inspired differential evolution algorithm for deep belief network," IEEE Transactions on Instrumentation and Measurement, vol. 69, no. 10, pp. 7319-7327, 2020.

[11] H. Zhao, H. Liu, J. Xu, and W. Deng, "Performance prediction using high-order differential mathematical morphology gradient spectrum entropy and extreme learning machine," IEEE Transactions on Instrumentation and Measurement, vol. 69, no. 7, pp. 4165-4172, 2019.

[12] H. Zhao, J. Zheng, J. Xu, and W. Deng, "Fault diagnosis method based on principal component analysis and broad learning system," IEEE Access, vol. 7, pp. 99263-99272, 2019.

[13] W. Deng, R. Yao, H. Zhao, X. Yang, and G. Li, "A novel intelligent diagnosis method using optimal LS-SVM with improved PSO algorithm," Soft Computing, vol. 23, no. 7, pp. 2445-2462, 2019.

[14] Q. Xiong, X. Zhang, W.-F. Wang, and Y. Gu, "A parallel algorithm framework for feature extraction of EEG signals on MPI," Computational and Mathematical Methods in Medicine, vol. 2020, Article ID 9812019, 10 pages, 2020.

[15] M. Wang and H. Chen, "Chaotic multi-swarm whale optimizer boosted support vector machine for medical diagnosis," Applied Soft Computing, vol. 88, Article ID 105946, 2020.

[16] G. Liu, W. Jia, M. Wang et al., "Predicting cervical hyperextension injury: a covariance guided sine cosine support vector machine," IEEE Access, vol. 8, pp. 46895-46908, 2020.

[17] J. Tu, A. Lin, H. Chen, Y. Li, and C. Li, "Predict the entrepreneurial intention of fresh graduate students based on an adaptive support vector machine framework," Mathematical Problems in Engineering, vol. 2019, Article ID 2039872, 16 pages, 2019.

[18] H. Huang, S. Zhou, X. Feng et al., "A new fruit fly optimization algorithm enhanced support vector machine for diagnosis of breast cancer based on high-level features," BMC Bioinformatics, vol. 20, no. 8, p. 290, 2019.

[19] J. Xu, X. Zhang, H. Chen et al., "Automatic analysis of microaneurysms turnover to diagnose the progression of diabetic retinopathy," IEEE Access, vol. 6, pp. 9632-9642, 2018. 
[20] C. Li, L. Hou, B. Y. Sharma et al., "Developing a new intelligent system for the diagnosis of tuberculous pleural effusion," Computer Methods and Programs in Biomedicine, vol. 153, pp. 211-225, 2018.

[21] Y. Wei, N. Ni, D. Liu et al., "An improved grey wolf optimization strategy enhanced SVM and its application in predicting the second major," Mathematical Problems in Engineering, vol. 2017, Article ID 9316713, 12 pages, 2017.

[22] L. Hu, F. Lin, H. Li et al., "An intelligent prognostic system for analyzing patients with paraquat poisoning using arterial blood gas indexes," Journal of Pharmacological and Toxicological Methods, vol. 84, pp. 78-85, 2017.

[23] H. Chen, L. Hu, H. Li et al., "An effective machine learning approach for prognosis of paraquat poisoning patients using blood routine indexes," Basic \& Clinical Pharmacology \& Toxicology, vol. 120, no. 1, pp. 86-96, 2017.

[24] Z. Cai, J. Gu, and H.-L. Chen, "A new hybrid intelligent framework for predicting Parkinson's disease," IEEE Access, vol. 5, pp. 17188-17200, 2017.

[25] L. Shen, H. Chen, Z. Yu et al., "Evolving support vector machines using fruit fly optimization for medical data classification," Knowledge-Based Systems, vol. 96, pp. 61-75, 2016.

[26] W.-L. Zuo, Z.-Y. Wang, T. Liu, and H.-L. Chen, "Effective detection of Parkinson's disease using an adaptive fuzzy $k$ nearest neighbor approach," Biomedical Signal Processing and Control, vol. 8, no. 4, pp. 364-373, 2013.

[27] H.-L. Chen, C.-C. Huang, X.-G. Yu et al., "An efficient diagnosis system for detection of Parkinson's disease using fuzzy $k$-nearest neighbor approach," Expert Systems with Applications, vol. 40, no. 1, pp. 263-271, 2013.

[28] Z. Cai, J. Gu, C. Wen et al., "An intelligent Parkinson's disease diagnostic system based on a chaotic bacterial foraging optimization enhanced fuzzy KNN approach," Computational and Mathematical Methods in Medicine, vol. 2018, Article ID 2396952, 24 pages, 2018.

[29] J. Zhu, X. Zhao, H. Li, H. Chen, and G. Wu, “An effective machine learning approach for identifying the glyphosate poisoning status in rats using blood routine test," IEEE Access, vol. 6, pp. 15653-15662, 2018.

[30] A. Lin, Q. Wu, A. A. Heidari et al., "Predicting intentions of students for master programs using a chaos-induced sine cosine-based fuzzy $K$-nearest neighbor classifier," IEEE Access, vol. 7, pp. 67235-67248, 2019.

[31] Y. Li, W.-G. Cui, H. Huang, Y.-Z. Guo, K. Li, and T. Tan, "Epileptic seizure detection in EEG signals using sparse multiscale radial basis function networks and the Fisher vector approach," Knowledge-Based Systems, vol. 164, pp. 96-106, 2019.

[32] Y. Li, J. Liu, Z. Tang, and B. Lei, "Deep spatial-temporal feature fusion from adaptive dynamic functional connectivity for MCI identification," IEEE Transactions on Medical Imaging, vol. 39, no. 9, pp. 2818-2830, 2020.

[33] Y. Li, Y. Liu, W.-G. Cui, Y.-Z. Guo, H. Huang, and Z.-Y. Hu, "Epileptic seizure detection in EEG signals using a unified temporal-spectral squeeze-and-excitation network," IEEE Transactions on Neural Systems and Rehabilitation Engineering, vol. 28, no. 4, pp. 782-794, 2020.

[34] Y. Wei, H. Lv, M. Chen et al., "Predicting entrepreneurial intention of students: an extreme learning machine with Gaussian barebone harris hawks optimizer," IEEE Access, vol. 8, pp. 76841-76855, 2020.

[35] W. Zhu, C. Ma, X. Zhao et al., "Evaluation of sino foreign cooperative education project using orthogonal sine cosine optimized kernel extreme learning machine," IEEE Access, vol. 8, pp. 61107-61123, 2020.

[36] H.-L. Chen, G. Wang, C. Ma, Z.-N. Cai, W.-B. Liu, and S.-J. Wang, "An efficient hybrid kernel extreme learning machine approach for early diagnosis of Parkinson's disease," Neurocomputing, vol. 184, pp. 131-144, 2016.

[37] X. Zhao, D. Li, B. Yang, S. Liu, Z. Pan, and H. Chen, “An efficient and effective automatic recognition system for online recognition of foreign fibers in cotton," IEEE Access, vol. 4, pp. 8465-8475, 2016.

[38] Y. Xu, H. Chen, A. A. Heidari et al., "An efficient chaotic mutative moth-flame-inspired optimizer for global optimization tasks," Expert Systems with Applications, vol. 129, pp. 135-155, 2019

[39] P. K. Wong, K. I. Wong, C. M. Vong, and C. S. Cheung, "Modeling and optimization of biodiesel engine performance using kernel-based extreme learning machine and cuckoo search," Renewable Energy, vol. 74, pp. 640-647, 2015.

[40] D. Zhao, C. Huang, Y. Wei, F. Yu, M. Wang, and H. Chen, "An effective computational model for bankruptcy prediction using kernel extreme learning machine approach," Computational Economics, vol. 49, no. 2, pp. 325-341, 2017.

[41] G. Y. Du, Q. Xu, and X. Y. Yang, "Fault diagnosis of rotating machinery components using a deep kernel extreme learning machine under different working conditions," Measurement Science and Technology, vol. 31, no. 11, Article ID 11590, 2020.

[42] X. B. Wang, X. Zhang, Z. Li, and J. Wu, "Ensemble extreme learning machines for compound-fault diagnosis of rotating machinery," Knowledge-Based Systems, vol. 188, Article ID 105012, 2020

[43] Y. Tian, X. Lei, X. Liu, M. Liu, X. Wen, and H. Wang, “A novel deep learning diagnosis scheme for rotating machinery using adaptive local iterative filtering and ensemble hierarchical extreme learning machine," Advances in Mechanical Engineering, vol. 11, no. 5, 2019.

[44] J.-H. Zhong, J. Zhang, J. Liang, and H. Wang, "Multi-fault rapid diagnosis for wind turbine gearbox using sparse bayesian extreme learning machine," IEEE Access, vol. 7, pp. 773-781, 2019.

[45] H. Lu, B. Du, J. Liu, H. Xia, and W. K. Yeap, "A kernel extreme learning machine algorithm based on improved particle swam optimization," Memetic Computing, vol. 9, no. 2, pp. 121-128, 2017.

[46] J. Luo, H. Chen, Q. Zhang, Y. Xu, H. Huang, and X. Zhao, "An improved grasshopper optimization algorithm with application to financial stress prediction," Applied Mathematical Modelling, vol. 64, pp. 654-668, 2018.

[47] X. Wang, Z. Wang, J. Weng, C. Wen, H. Chen, and X. Wang, "A new effective machine learning framework for sepsis diagnosis," IEEE Access, vol. 6, pp. 48300-48310, 2018.

[48] Y. Tian, J. Zhang, L. Chen, Y. Geng, and X. Wang, "Single wearable accelerometer-based human activity recognition via kernel discriminant analysis and QPSO-KELM classifier," IEEE Access, vol. 7, pp. 109216-109227, 2019.

[49] S. K. Baliarsingh, S. Vipsita, K. Muhammad, B. Dash, and S. Bakshi, "Analysis of high-dimensional genomic data employing a novel bio-inspired algorithm," Applied Soft Computing, vol. 77, pp. 520-532, 2019.

[50] W. Hu, H. Chang, and X. Gu, "A novel fault diagnosis technique for wind turbine gearbox," Applied Soft Computing, vol. 82, Article ID 105556, 2019. 
[51] A. K. Pani and N. Nayak, "Forecasting solar irradiance with weather classification and chaotic gravitational search algorithm based wavelet kernel extreme learning machine," International Journal of Renewable Energy Research, vol. 9, no. 4, pp. 1650-1659, 2019.

[52] J. Luo, H. Chen, Z. Hu et al., "A new kernel extreme learning machine framework for somatization disorder diagnosis," IEEE Access, vol. 7, pp. 45512-45525, 2019.

[53] Q. Li, H. Chen, H. Huang et al., "An enhanced grey wolf optimization based feature selection wrapped kernel extreme learning machine for medical diagnosis," Computational and Mathematical Methods in Medicine, vol. 2017, Article ID 9512741, 15 pages, 2017.

[54] R. Bisoi, P. K. Dash, and A. K. Parida, "Hybrid variational mode decomposition and evolutionary robust kernel extreme learning machine for stock price and movement prediction on daily basis," Applied Soft Computing, vol. 74, pp. 652-678, 2019.

[55] M. Wang, H. Chen, B. Yang et al., "Toward an optimal kernel extreme learning machine using a chaotic moth-flame optimization strategy with applications in medical diagnoses," Neurocomputing, vol. 267, pp. 69-84, 2017.

[56] M. Wang, H. Chen, H. Li et al., "Grey wolf optimization evolving kernel extreme learning machine: application to bankruptcy prediction," Engineering Applications of Artificial Intelligence, vol. 63, pp. 54-68, 2017.

[57] A. A. Heidari, R. A. Abbaspour, and H. Chen, "Efficient boosted grey wolf optimizers for global search and kernel extreme learning machine training," Applied Soft Computing, vol. 81, Article ID 105521, 2019.

[58] H. Chen, Q. Zhang, J. Luo, Y. Xu, and X. Zhang, "An enhanced bacterial foraging optimization and its application for training kernel extreme learning machine," Applied Soft Computing, vol. 86, Article ID 105884, 2020.

[59] L. M. Wang and Y. M. Shao, "Fault feature extraction of rotating machinery using a reweighted complete ensemble empirical mode decomposition with adaptive noise and demodulation analysis," Mechanical Systems and Signal Processing, vol. 138, Article ID 106545, 2020.

[60] F. F. Chen, M. Cheng, B. Tang, W. Xiao, B. Chen, and X. Shi, "A novel optimized multi-kernel relevance vector machine with selected sensitive features and its application in early fault diagnosis for rolling bearings," Measurement, vol. 156, Article ID 107583, 2020.

[61] M. Xiao, C. Zhang, K. Wen, L. Xiong, G. Geng, and D. Wu, "Bearing fault feature extraction method based on complete ensemble empirical mode decomposition with adaptive noise," Journal of Vibroengineering, vol. 20, no. 7, pp. 2622-2631, 2018.

[62] L. L. Jiang, H. Tan, X. Li, L. Chen, and D. Yang, "CEEMDANbased permutation entropy: a suitable feature for the fault identification of spiral-bevel gears," Shock and Vibration, vol. 2019, Article ID 7806015, 13 pages, 2019.

[63] X. Peng, J. Li, G. Wang et al., "Random forest based optimal feature selection for partial discharge pattern recognition in HV cables," IEEE Transactions on Power Delivery, vol. 34, no. 4, pp. 1715-1724, 2019.

[64] B. Cao, J. Zhao, Z. Lv, Y. Gu, P. Yang, and S. K. Halgamuge, "Multiobjective evolution of fuzzy rough neural network via distributed parallelism for stock prediction," IEEE Transactions on Fuzzy Systems, vol. 28, no. 5, pp. 939-952, 2020.

[65] B. Cao, J. Zhao, P. Yang et al., "Multiobjective 3-D topology optimization of next-generation wireless data center network," IEEE Transactions on Industrial Informatics, vol. 16, no. 5, pp. 3597-3605, 2019.

[66] B. Cao, J. Zhao, Y. Gu, Y. Ling, and X. Ma, “Applying graphbased differential grouping for multiobjective large-scale optimization," Swarm and Evolutionary Computation, vol. 53, Article ID 100626, 2020.

[67] B. Cao, S. Fan, J. Zhao, P. Yang, K. Muhammad, and M. Tanveer, "Quantum-enhanced multiobjective large-scale optimization via parallelism," Swarm and Evolutionary Computation, vol. 57, Article ID 100697, 2020.

[68] B. Cao, W. Dong, Z. Lv, Y. Gu, S. Singh, and P. Kumar, "Hybrid microgrid many-objective sizing optimization with fuzzy decision," IEEE Transactions on Fuzzy Systems, vol. 28, no. 11, pp. 2702-2710, 2020.

[69] B. Cao, X. Wang, W. Zhang, H. Song, and Z. Lv, "A manyobjective optimization model of industrial internet of things based on private blockchain," IEEE Network, vol. 34, no. 5, pp. 78-83, 2020.

[70] S. Qu, Y. Han, Z. Wu et al., "Consensus modeling with asymmetric cost based on data-driven robust optimization," Group Decision and Negotiation, pp. 1-38, 2020.

[71] X. Fu, P. Pace, G. Aloi, L. Yang, and G. Fortino, "Topology optimization against cascading failures on wireless sensor networks using a memetic algorithm," Computer Networks, vol. 177, Article ID 107327, 2020.

[72] H. Chen, H. Qiao, L. Xu, Q. Feng, and K. Cai, “A fuzzy optimization strategy for the implementation of RBF LSSVR model in Vis-NIR analysis of pomelo maturity," IEEE Transactions on Industrial Informatics, vol. 15, no. 11, pp. 5971-5979, 2019.

[73] S. Arora and S. Singh, "Butterfly algorithm with levy flights for global optimization," in Proceedings of the 2015 International Conference on Signal Processing, Computing and Control, pp. 220-224, Solan, India, September 2015.

[74] G.-G. Wang, "Moth search algorithm: a bio-inspired metaheuristic algorithm for global optimization problems," Memetic Computing, vol. 10, no. 2, pp. 151-164, 2018.

[75] G.-B. Huang, H. Zhou, X. Ding, and R. Zhang, "Extreme learning machine for regression and multiclass classification," IEEE Transactions on Systems Man and Cybernetics Part B-Cybernetics, vol. 42, no. 2, pp. 513-529, 2012.

[76] Z. Lei, Y. Zhou, B. Sun, and W. Sun, "An intrinsic timescale decomposition-based kernel extreme learning machine method to detect tool wear conditions in the milling process," International Journal of Advanced Manufacturing Technology, vol. 106, no. 3-4, pp. 1203-1212, 2020.

[77] X. F. Long, P. Yang, H. Guo, Z. Zhao, and X. Wu, "A CBAKELM-based recognition method for fault diagnosis of wind turbines with time-domain analysis and multisensor data fusion," Shock and Vibration, vol. 2019, Article ID 7490750, 14 pages, 2019.

[78] Z. J. Wang, L. Zheng, J. Wang, and W. Du, "Research on novel bearing fault diagnosis method based on improved krill herd algorithm and kernel extreme learning machine," Complexity, vol. 2019, Article ID 4031795, 19 pages, 2019.

[79] X.-S. Yang, "A new metaheuristic bat-inspired algorithm," in Nature Inspired Cooperative Strategies for Optimization (NICSO 2010), J. R. González et al., Ed., Springer, Berlin, Germany, pp. 65-74, 2010.

[80] S. Mirjalili, "SCA: a sine cosine algorithm for solving optimization problems," Knowledge-Based Systems, vol. 96, pp. 120-133, 2016. 
[81] S. Mirjalili and A. Lewis, "The whale optimization algorithm," Advances in Engineering Software, vol. 95, pp. 51-67, 2016.

[82] Y. Cao, Y. Li, G. Zhang, K. Jermsittiparsert, and M. Nasseri, "An efficient terminal voltage control for PEMFC based on an improved version of whale optimization algorithm," Energy Reports, vol. 6, pp. 530-542, 2020.

[83] H. Nenavath, D. R. Kumar Jatoth, and D. S. Das, "A synergy of the sine-cosine algorithm and particle swarm optimizer for improved global optimization and object tracking," Swarm and Evolutionary Computation, vol. 43, pp. 1-30, 2018.

[84] H. Nenavath and R. K. Jatoth, "Hybridizing sine cosine algorithm with differential evolution for global optimization and object tracking," Applied Soft Computing, vol. 62, pp. 1019-1043, 2018.

[85] N. Kumar, I. Hussain, B. Singh, and B. K. Panigrahi, "Single sensor-based MPPT of partially shaded PV system for battery charging by using cauchy and Gaussian sine cosine optimization," IEEE Transactions on Energy Conversion, vol. 32, no. 3, pp. 983-992, 2017.

[86] B. R. Adarsh, T. Raghunathan, T. Jayabarathi, and X.-S. Yang, "Economic dispatch using chaotic bat algorithm," Energy, vol. 96, pp. 666-675, 2016.

[87] N. Khashan, M. A. El-Hosseini, M. Badawy, and A. Y. Haikal, "Biped robot stability based on an A-C parametric whale optimization algorithm," Journal of Computational Science, vol. 31, 2018.

[88] G. Kaur and S. Arora, "Chaotic whale optimization algorithm," Journal of Computational Design and Engineering, vol. 5, no. 3, pp. 275-284, 2018.

[89] K. Shi, J. Wang, Y. Tang, and S. Zhong, "Reliable asynchronous sampled-data filtering of T-S fuzzy uncertain delayed neural networks with stochastic switched topologies," Fuzzy Sets and Systems, vol. 381, pp. 1-25, 2020.

[90] K. Shi, J. Wang, S. Zhong, Y. Tang, and J. Cheng, "Nonfragile memory filtering of T-S fuzzy delayed neural networks based on switched fuzzy sampled-data control," Fuzzy Sets and Systems, vol. 394, pp. 40-64, 2020.

[91] K. Shi, Y. Tang, S. Zhong, C. Yin, X. Huang, and W. Wang, "Nonfragile asynchronous control for uncertain chaotic Lurie network systems with Bernoulli stochastic process," International Journal of Robust and Nonlinear Control, vol. 28, no. 5, pp. 1693-1714, 2018.

[92] S. Yang, B. Deng, J. Wang et al., "Scalable digital neuromorphic architecture for large-scale biophysically meaningful neural network with multi-compartment neurons," IEEE Transactions on Neural Networks and Learning Systems, vol. 31, no. 1, pp. 148-162, 2019.

[93] H. Zhang, Z. Wang, W. Chen et al., "Ensemble mutationdriven salp swarm algorithm with restart mechanism: framework and fundamental analysis," Expert Systems with Applications, vol. 165, Article ID 113897, 2021.

[94] T. Ni, H. Chang, T. Song et al., "Non-intrusive online distributed pulse shrinking-based interconnect testing in $2.5 \mathrm{D}$ IC," IEEE Transactions on Circuits and Systems II: Express Briefs, vol. 67, no. 11, pp. 2657-2661, 2020.

[95] H. Zhang, Z. Qiu, J. Cao, M. Abdel-Aty, and L. Xiong, "Event-triggered synchronization for neutral-type semimarkovian neural networks with partial mode-dependent time-varying delays," IEEE Transactions on Neural Networks and Learning Systems, vol. 31, no. 11, pp. 4437-4450, 2020.
[96] Z. Lv and L. Qiao, "Deep belief network and linear perceptron based cognitive computing for collaborative robots," Applied Soft Computing, vol. 92, Article ID 106300, 2020.

[97] J. Liu, C. Wu, G. Wu, and X. Wang, "A novel differential search algorithm and applications for structure design," Applied Mathematics and Computation, vol. 268, pp. 246269, 2015.

[98] G. Sun, B. Yang, Z. Yang, and G. Xu, "An adaptive differential evolution with combined strategy for global numerical optimization," Soft Computing, vol. 23, no. 3, pp. 1-20, 2019.

[99] J. Derrac, S. García, D. Molina, and F. Herrera, "A practical tutorial on the use of nonparametric statistical tests as a methodology for comparing evolutionary and swarm intelligence algorithms," Swarm and Evolutionary Computation, vol. 1, no. 1, pp. 3-18, 2011.

[100] J. Xia, H. Chen, Q. Li et al., "Ultrasound-based differentiation of malignant and benign thyroid Nodules: an extreme learning machine approach," Computer Methods and Programs in Biomedicine, vol. 147, pp. 37-49, 2017.

[101] Z. Cai, J. Gu, and H.-L. Chen, "A new hybrid intelligent framework for predicting Parkinson's disease," IEEE Access, vol. 5, pp. 17188-17200, 2017.

[102] L. Hu, H. Li, Z. Cai et al., "A new machine-learning method to prognosticate paraquat poisoned patients by combining coagulation, liver, and kidney indices," PLoS One, vol. 12, no. 10, Article ID e0186427, 2017.

[103] C. Wen, F. Lin, B. Huang et al., "Metabolomics analysis in acute paraquat poisoning patients based on UPLC-Q-TOFMS and machine learning approach," Chemical Research in Toxicology, vol. 32, no. 4, pp. 629-637, 2019.

[104] J. Zhu, F. Zhu, S. Huang et al., "A new evolutionary machine learning approach for identifying pyrene induced hepatotoxicity and renal dysfunction in rats," IEEE Access, vol. 7, pp. 15320-15329, 2019.

[105] Y. Xu, K. Yu, P. Wang, H. Chen, X. Zhao, and J. Zhu, “A new hybrid machine learning approach for prediction of phenanthrene toxicity on mice," IEEE Access, vol. 7, pp. 138461-138472, 2019.

[106] G. Wang, W.-Y. Zhang, Q. Ning, and H.-L. Chen, "A novel framework based on ACO and PSO for RNA secondary structure prediction," Mathematical Problems in Engineering, vol. 2013, Article ID 796304, 8 pages, 2013.

[107] G. Liu, W. Jia, Y. Luo et al., "Prediction optimization of cervical hyperextension injury: kernel extreme learning machines with orthogonal learning butterfly optimizer and broyden- fletcher-goldfarb-shanno algorithms," IEEE Access, vol. 8, pp. 119911-119930, 2020.

[108] T. Liu, L. Hu, C. Ma, Z.-Y. Wang, and H.-L. Chen, “A fast approach for detection of erythemato-squamous diseases based on extreme learning machine with maximum relevance minimum redundancy feature selection," International Journal of Systems Science, vol. 46, no. 5, pp. 919-931, 2015.

[109] D. Liu, S. Wang, D. Huang, G. Deng, F. Zeng, and H. Chen, "Medical image classification using spatial adjacent histogram based on adaptive local binary patterns," Computers in Biology and Medicine, vol. 72, pp. 185-200, 2016.

[110] X. Fei, J. Wang, S. Ying, Z. Hu, and J. Shi, "Projective parameter transfer based sparse multiple empirical kernel learning machine for diagnosis of brain disease," Neurocomputing, vol. 413, pp. 271-283, 2020.

[111] Y. Liu, Y. Chang, C. Zhang et al., "Influence of mRNA features on siRNA interference efficacy," Journal of 
Bioinformatics and Computational Biology, vol. 11, no. 3, Article ID 1341004, 2013.

[112] C. Wu, P. Wu, J. Wang, R. Jiang, M. Chen, and X. Wang, "Critical review of data-driven decision-making in bridge operation and maintenance," Structure and Infrastructure Engineering, pp. 1-24, 2020.

[113] S. Liu, W. Yu, F. T. S. Chan, and B. Niu, "A variable weightbased hybrid approach for multi-attribute group decision making under interval-valued intuitionistic fuzzy sets," International Journal of Intelligent Systems, vol. 36, no. 2, pp. 1015-1052, 2021.

[114] S. Liu, F. T. S. Chan, and W. Ran, "Decision making for the selection of cloud vendor: an improved approach under group decision-making with integrated weights and objective/subjective attributes," Expert Systems with Applications, vol. 55, pp. 37-47, 2016.

[115] C.-W. Zhang, J.-P. Ou, and J.-Q. Zhang, "Parameter optimization and analysis of a vehicle suspension system controlled by magnetorheological fluid dampers," Structural Control and Health Monitoring, vol. 13, no. 5, pp. 885-896, 2006.

[116] T. Qiu, X. Shi, J. Wang et al., "Deep learning: a rapid and efficient route to automatic metasurface design," Advanced Science, vol. 6, no. 12, Article ID 1900128, 2019.

[117] T. Li, M. Xu, C. Zhu, R. Yang, Z. Wang, and Z. Guan, “A deep learning approach for multi-frame in-loop filter of HEVC," IEEE Transactions on Image Processing, vol. 28, no. 11, pp. 5663-5678, 2019.

[118] H. Chen, A. Chen, L. Xu et al., "A deep learning CNN architecture applied in smart near-infrared analysis of water pollution for agricultural irrigation resources," Agricultural Water Management, vol. 240, Article ID 106303, 2020.

[119] D. Zhao, L. Liu, F. Yu et al., "Ant colony optimization with horizontal and vertical crossover search: fundamental visions for multi-threshold image segmentation," Expert Systems with Applications, Article ID 114122, 2020.

[120] D. Zhao, L. Liu, F. Yu et al., "Chaotic random spare ant colony optimization for multi-threshold image segmentation of 2D Kapur entropy," Knowledge-Based Systems, Article ID 106510, 2020.

[121] S. Lu, X. Jin, H. Zhao, and Y. Zhao, "Real-time image marbleization," Multimedia Tools and Applications, vol. 64, no. 3, pp. 795-808, 2013.

[122] H.-L. Zhao, G.-Z. Nie, X.-J. Li, X.-G. Jin, and Z.-G. Pan, "Structure-aware nonlocal optimization framework for image colorization," Journal of Computer Science and Technology, vol. 30, no. 3, pp. 478-488, 2015.

[123] H. Zhao, H. Zhang, and X. Jin, "Efficient image decolorization with a multimodal contrast-preserving measure," Computers \& Graphics, vol. 70, pp. 251-260, 2018.

[124] H. Zhao, H. Guo, X. Jin, J. Shen, X. Mao, and J. Liu, "Parallel and efficient approximate nearest patch matching for image editing applications," Neurocomputing, vol. 305, pp. 39-50, 2018.

[125] Y. Zhang, R. Liu, A. A. Heidari et al., "Towards augmented kernel extreme learning models for bankruptcy prediction: algorithmic behavior and comprehensive analysis," Neurocomputing, 2020.

[126] S.-J. Wang, H.-L. Chen, W.-J. Yan, Y.-H. Chen, and X. Fu, "Face recognition and micro-expression recognition based on discriminant tensor subspace analysis plus extreme learning machine," Neural Processing Letters, vol. 39, no. 1, pp. 25-43, 2014
[127] X. Zhang, Y. Wang, X. Chen et al., "Decentralized adaptive neural approximated inverse control for a class of large-scale nonlinear hysteretic systems with time delays," IEEE Transactions on Systems, Man, and Cybernetics: Systems, vol. 49, no. 12, pp. 2424-2437, 2018.

[128] Z. Chen, M. Lu, Y. Zhou, and C. Chen, "Information synergy entropy based multi-feature information fusion for the operating condition identification in aluminium electrolysis," Information Sciences, vol. 548, pp. 275-294, 2021.

[129] X. Xue, S. Wang, L. Zhang, Z. Feng, and Y. Guo, "Social learning evolution (SLE): computational experiment-based modeling framework of social manufacturing," IEEE Transactions on Industrial Informatics, vol. 15, no. 6, pp. 3343-3355, 2019

[130] R. Guan, H. Zhang, Y. Liang, F. Giunchiglia, L. Huang, and $\mathrm{X}$. Feng, "Deep feature-based text clustering and its explanation," IEEE Transactions on Knowledge and Data Engineering, p. 1, 2020.

[131] Y. Wang, J. See, Y.-H. Oh et al., "Effective recognition of facial micro-expressions with video motion magnification," Multimedia Tools and Applications, vol. 76, no. 20, pp. 21665-21690, 2017.

[132] C. Yang, L. Bruzzone, H. Zhao, Y. Tan, and R. Guan, "Superpixel-based unsupervised band selection for classification of hyperspectral images," IEEE Transactions on Geoscience and Remote Sensing, vol. 56, no. 12, pp. 72307245, 2018.

[133] J. Tu, A. Lin, H. Chen, Y. Li, and C. Li, "Predict the entrepreneurial intention of fresh graduate students based on an adaptive support vector machine framework," Mathematical Problems in Engineering, vol. 2019, Article ID 2039872, 16 pages, 2019.

[134] H. Tang, Y. Xu, A. Lin et al., "Predicting green consumption behaviors of students using efficient firefly grey wolf-assisted $K$-nearest neighbor classifiers," IEEE Access, vol. 8, pp. 35546-35562, 2020. 